\title{
ASPECTOS EM TEORIAS DE CAMPOS A TEMPERATURA FINITA
}

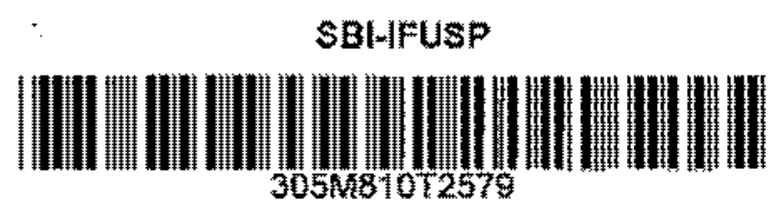

Orientador: Prof. Dr. Josif Frenkel

Comissão Examinadora:

Prof. Dr. Josif Frenkel 40 it tuke

Prof. Dr. Marcelo O. Caninha Gomes

Prof. Dr. Carlos Ourivio Escobar

Prof. Dr. Abraham H. Zimerman

Prof. Dr. João Barcelos Neto

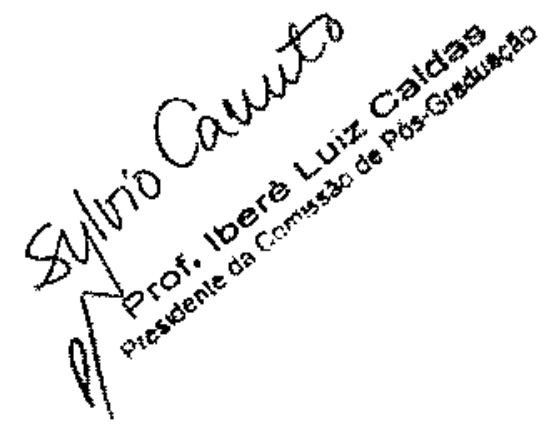

SÃO PAULO

1996

\begin{abstract}
André Pereira de Almeida
Tese de doutorado apresentada ao Instituto de Fisica da Universidade de Săo Paulo para a obtenção do título de Doutor em Ciências.
\end{abstract}




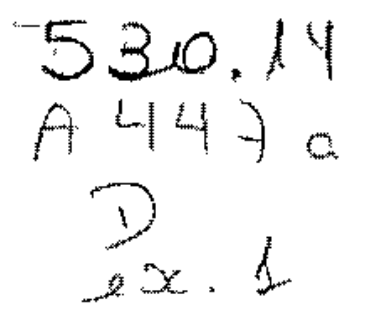

\section{FICHA CATALOGRÁFICA}

Preparada pelo Serviço de Biblioteca e Informação do Instituto de Física da Universidade de São Paulo

\section{Almeida, Andié Pereira de}

Aspectos em Teorias de Campos à Temperatura Finita. São Paulo, 1997.

Tese (Doutoramento) Universidade de São Paulo. Instituto de Física - Departamento de Fisica Matemática Area de Concentração: Teoria de Campos \& Particulas Orientador: Prof. Dr. Josif Frenkel

Unitermos: 1. Teoria de Campos; 2. Cromodinâmica Quântica; 3. Temperatura Finita. 


\section{Resumo}

Este trabalho estuda o comportamento de algumas teonits de campos quando a temperatura năo é mula. O comportamento infravernelho destas teorias é, em alguns casos, extremamente complicado. No contexto da aproximaçäo ladder, estudanos o cancelamento das divergências infravermelhas para a pressäo na teoria escalar com acoplamento $\lambda \phi^{3}$ e na teoria de Yang-Mills. Nos provamos que as divergências infravermelhas destas teorias que aparecem na série perturbativa se cancelam na aproximaçäo ladder. As massas tếrmicas que sấo de fundanental importância para a compreensảo destes comportaroentos säo tambern estudadas no contexto da gravitaçăo. Nós calcularnos algumas propriedades do tensor de polarização do gráviton, em particular cajculamos a massa de Jeans que descreve a instabilidade da teoria a temperatura finita. 


\begin{abstract}
This work studies the behaviour of some fields theories with non-zero temperatures. The infrared behaviour of these theories is, in some cases, quite complicated. In the context of the ladder approximation, we study the cancellation of infrared singularities of the pressure of the scalar theory with a $\lambda \phi^{3}$ coupling and of the Yang Mills theory. We prove that the infrared divergencies of these theories that appear in the perturbative expansion cancels out in the ladder approximation. The thermal masses, which are of fundamental importance to understand these behaviours are also studied in the context of gravity. We derive some properties of the graviton's propaw gator, in particular, we have calculated the Jeans' mass which describe the instubility of this theory at finite temperature.
\end{abstract}




\section{Agradecimentos}

Agradeço à minha esposa, Ana Cristina Granado Pereira de Alrneida, pelo apoio; ao meu orientador, Josif Frenkel, pela paciência, e à FAPESP pela ajuda financeira. 


\section{Conteúdo}

1 Fundamentos da teoria de campos a temperatura finita 4

2 Divergências Infravermelhas em tearias de campo a temperatura finita

3 Estudo do compor tamento infravermelho na aproximaçäo "ladder"

4 Efeitos térmicos em um teoria de campos em espaço curvo 29

5 Efeitos de campos térmicos no tensor de polarizaçäo do gráviton

Apendice A 


\section{Introdução}

O objetivo de teorias de carapo a temperatura finita é entender o comportamento da matéria quando a temperatura é extremamente elevada, a tal ponto que os quarks se desconfinam, ou quando a densidade é tão alta que os prótons, nêutrons ou hádrons em geral se sobrepòem, formando um plasma de quarks e glúons. Tais condiçoses extremas ocorrem somente muito longe da experiência humana diária, mas sāo de importância fundarnental para a compreensão de processos que aconteceram no principio do universo, que o tornaram tal como o conhecemos hoje, ou para o estudo do que acontece no interior de urna estrela de nêutrons onde a matéria nuclear se funde, ou ainda para estudarmos colisōes altamente energéticas de núcleos pesados. Para estes estudos precisamos compreender os efeitos coletivos gerados pela associaçâo da teoria de campos usual com a termodinârnica.

Um dos campos mais interessantes da teoria de campos a temperatura finita é o estudo da teoria da interaçäo dos quarks e glíons, a cronodinânaica quântica. Acredita-se que os quarks, a temperatura zero, permanecem confinados. A medida em que temperatura aurnenta, porén, os hadrons devem aumentar de tamanho, pois a energia interna dos quarks aumenta e, a uma deterninada temperatura crítica, os quarks devem se desconfinar formando um plasrna de quarks e glúnons livres. No entanto, o estudo desta teoria é extremamente complicado, pois as divergências infravermelhas que já aparecem a temperatura zero tornam esta teoria impossivel de se calcular na prática. Esta divergência, que está intimamente ligada ao fato de os gluons näo terem massa, tornam a teoria de perturbaçôes inaplicável alérn de uma determinada ordem na constante de acoplamento, pois as divergências tornam-se cada yez mais severas. Em alguns modelos, como no caso de uma teoria escalar com acoplamento $\lambda \phi^{3}{ }_{\text {, }}$ este problema näo aparece a temperatura finita pois as particulas näo massivas ganham unna massa têrmica que surge naturalmente 
da teoria quando a temperatura näo é nula, e esta massa permite eliminar todas as divergências irufravermelhas que venham a surgir[l]. Infelizmente isto é apenas em parte aplicável a QCD, pois enquanto os gluons referentes a parte cromoelétrica da teoria efetivamente ganham massa térnica, os gluons cromomagneticos năo apresentam uma massa deste tipo, impossibilitando assim um tratamento simples da teoria.

Bntretanto, para que a teoria faça sentido é de se esperar que grandezas físicas mensurâyeis como a pressão estejam de alguma forma livres destas divergencias, ou seja, que elas se cancelem em um tratamento não perturbativo. A primeira parte desta tese é voltada este importante problema. Aqu é proposto um mecanismo pelo qual estas divergencias podem se cancelar, estudando um modelo simplificado das equaçós de Schwinger-Dyson para a cromodinàmica. As equaçōes de Schwinger-Dyson săo equaçōes näo perturbativas que a teoria deve obedecer. Estas equaçones, no entanto, sâo extremamente complicadas e, em geral, säo um conjunto infinito de equaçōes; o que as tornam impossiveis de lidar. Assim, temos que, de alguma forma, truncar estas equaçōes. Para isto usamos a chamada aproximaçāo "ladder" que consiste em desprezar a contribuiçăo das funçōes de Green de três e quatro pontos do glúon, de modo semelhante ao proposto por Kajantie e Kapusta[2]. Esta aproximaçấo ja foi utilizada por Mandelstam[3] para entender como pode ocorrer o confmaraento a temperatura zero, e por Jackiw a Templeton[4] para mostrar como teorias super-renormalizáveis curam as divergências ultravioletas. Com esta aproximaçắ possível obter um conjunto finito de equaçoes tentar resolva que, apesar de que todos os termos perturbativos divergern a partir de uma determinada ordern de perturbaçăo, o resultado fnal é finito. Este trabalho foi publicado na Physical Review 5 .

$O$ conceito de massas termicas desempenham tambéro um papel funda* mental em outro campo desta teoria, a gravitaçäo a temperatura finita. Em gravitação é notória a diffculdade de se obter uma teoria quântica convin cente, porém podemos estudar a gravitaçäo quando esta entra de uma forma semi-clássica, no estudo de teorias de campos em espaços curvo. À ternperatura finita isto é possivel se supusermos que a temperatura do plasma está bem abaixo da escala de Planck, isto é, após os primeiros segundos da formação do universo. Neste regime, podemos obter uma teoria consistente para um plasma sob açäo de um campo gravitacional. Um resultado expresssivo desta teoria é que o grúkiton apresenta uma massa térmica imaginćria. 
para um determinado modo de vibração do plasma. Isto identiffca uma instabilidade do espaço plano, indicando que a qualcquer temperatura näo nula, o espaço plano não é o estado de mínima energia e vai decair. Este resultado é bem conhecido e foi obtido pela primeira vez por sir James Jeans em 1902 para um fluido sob ação de um campo gravitacional newtoniano. O significado deste resultado é simples: a matéria espalhada uniformenente por uma região grande do espaço é instável, assim ela tende a se fragmentar formando as galáxias tais como as conkecernos hoje. O estudio do propar gador do gráviton desempenha, desta forma, um ponto fundamental para entendermos esta teoria. Nesta tese obtivernos erte propagador, para o caso de uma partíula escalar conforme sem massa, de forma fechada. Obtivemos também que ele apresenta três modos de vibração distintos, um associado a una massa imaginária, análoga à massa de Jeans clässica; outro modo associado a uma massa real que atenua as vibraçöes do plasma, e um terceiro modo que não apresenta massa, análogo ì parte magnética e uma teoria e Yang-Mills. O estudo deste propagador que consta nesta tese foi também publicado na Physical Revievi[6].

Esta tese está organizada da seguinte forma: no prixneiro capítulo apresento um estudo de teorias de campos a temperatura finita, explicando basicamente as ferramentas teóricas necessárias para compreensão do resto da tese. Para mais detalhes recomendo a referência [7]. O segundo capítulo trata basicamente da colocacão do problema das divergências infravermelhas numa teoria de Yang-Mills a temperatura finita, como elas aparecern e quais as difculdades que surgem partir delas. $O$ terceiro capítulo é uma análise de cono estas divergêneias se cancelam na aproximação ladder. O quarto capittulo descrevo alguns pontos de teorias de campos sobra espaço curvo. Para maiores detalhes recomendo os livros [8] e [9]. Finalmente, no kiltimo capítulo estudo como deve se comportar o tensor de polarizaçăo do gráviton, calculo de forma fechada este tensor e analiso o surgimento de massas térmicas neste propagador. 


\section{Capítulo 1}

\section{Fundamentos da teoria de campos a temperatura finita}

Vamos considerar um sistema descrito por um operador hamiltoniano $\hat{H}$ em contato com um reservatório de calor a temperatura $T=1 / \beta$, isto é, o chamado ensamble canônico. Neste sistema a matriz densidade estatística é expressa da forma:

$$
\rho=\exp (-\beta \hat{H})
$$

e, a partir dela, podemos calcular a média estatística de um operador $\hat{A}$ :

$$
A=\frac{\operatorname{Tr} \rho \hat{A}}{\operatorname{Tr} \rho}
$$

O ponto de partida para estudarmos os efeito térmicos de uma teoria é a funçào partição:

$$
Z=\operatorname{Tr} \rho
$$

A partir dela podemos obter todas as propriedades térmicas relevantes do sistema, tais como a pressão, energia ou entropia. Em teoria de campos a função partiçāo pode ser definida como:

$$
Z=\int \mathcal{D} \phi<\phi|\exp (-\beta \hat{H})| \phi>
$$

onde estamos integrando sobre todos os estados possíveis dos campos envolvidos no sistema. 
Por outro lado, sabemos da teoria quântica de campos que:

$$
<\phi_{a}\left|\exp \left(i t_{1} \hat{H}\right)\right| \phi_{b}>
$$

é a amplitude de probabilidade de transiçäo de um estado $\phi_{a}$ para um estado $\phi_{b}$ em um tempo $t_{1}$. Segundo o teorema de Kac-Feynman esta fórmula pode ser escrita na forma de uma integral funcional:

$$
\int_{\phi_{0}(t=0)}^{\phi_{b}\left(t=t_{1}\right)} \mathcal{D} \phi \exp i S
$$

onde

$$
S=\int_{0}^{t_{1}} d t \int d^{3} \pi \mathcal{L}_{\text {eff }}(\phi)
$$

a ação do sistema neste intervalo de tempo e $\mathcal{L}_{\text {eff }}$ é a densidade lagrangeana efetiva do sistema. $O$ aparecimento da lagrangeana efetiva em lugar da lagrangeana usual se deve a que, exo teorias de sistemas vinculados, necessitamos de termos de fixaçăo de gauge para obternos o valor correto da integral funcional, como é bem conhecido de teorias quânticas de campos de gauge.

A semelhança das fốrnulas (1.4) e (1.5) é clara. Logo gostariamos de es-

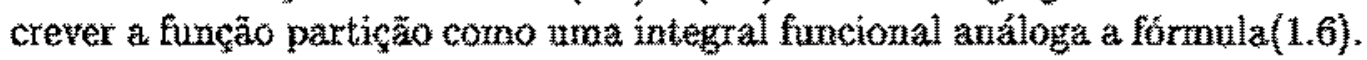
Para isso observarnos que fazendo a substuiçäo $\beta \rightarrow-$ it $_{1} \mathrm{ern}(1.4)$ obteremos (1.5) integrado sobre todos os estados iniciais e finais iguais, isto é integrado sobre todas as funçöes periodicas em $\hat{t}_{t}$ coro periodo $\beta$. Por esse motivo é de se esperar que a fiuç̧âo partiçăo possa ser escrita da forma:

$$
Z=\int_{\phi \text { peribdico }} D \phi \exp (-S)
$$

com

$$
S=\int_{0}^{B} d r \int d^{3} x \mathcal{L}_{\text {eff }}(\phi)
$$

Note que fizenos uma rotaçăo de $t \rightarrow \hat{t} \tau$ no plano complexo (urna rotaçāo de Wick), tornando o ternpo imagizário, isto é, fazendo uma continuaçäo

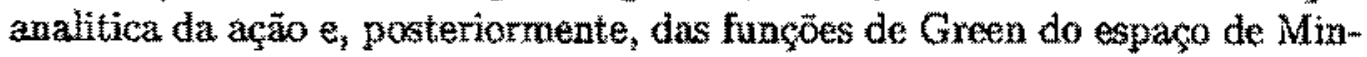
kowsky para o espaço Euclidiano. Por este motivo este método é chamado de forma jismo de tempo imaginário. $\hat{\mathrm{E}}$ interessante observar que, quando impomos que os campos sejam periódicos no tempo, quebramos parcialmente a 
simetria de Lorenz da teoria, o que era de se esperar, pois agora nós temos um referencial privilegiado, o referencial de repouso do plasma.

Como é bem conhecido, nós sabemos resolver exatamente apenas integrais funcionais de funçōes gaussianas, que correspondem a particulas livres, mas podemos usar a teoria de perturbaçōes no termo de acoplamento. A teoria de perturbaçōes em uma teoria de campo a ternperatura finita é bam sicamente análoga a teoria a temperatura zero, obtendo-se as grátícos as regras Feynman iguais ao caso de temperatura zero, porém como estarnos integrando apenas sobre funçöes periódicas no tempo, isto $\mathrm{E}$, funçôs que podem ser descritas por uma série de Fourrier no termpo, as componentes temporais do momento das partículas internas de um gráfico de Feynman só poderão ter valores discretos $k_{0}=2 \pi i n T \operatorname{com} n$ sendo urn número inteiro, como veremos.

Para prosseguirmos vamos estudar a principio a teoria escalar sern massa corn acoplamento $\lambda \phi^{3}$ en $d$ dimensôes definida pela dexsidade lagrangeana:

$$
\mathcal{L}=\frac{1}{2} \partial_{k} \phi \partial^{\mu} \phi+\frac{1}{6} \lambda \phi^{3}
$$

Em 6 dimensões sta teoria é renormalizavel e o acoplamento se torna adimensional. Esta teoria um bom modelo para entendermos a cromodinâmica quantica (QOD), pois, como esta, a teoria escalar é assintoticumente livre, tendo basicamente as mesmas propriedades da QCD sem as complicações desta. Infelimente esta teoria näo e bem definida globalmente, poís a lagrangeana nata 6 limitada inferiormente, como êcil de ver pelo termo de acoplamento, mas este problema näo aparece no tratamento pertubativo que memos usar.

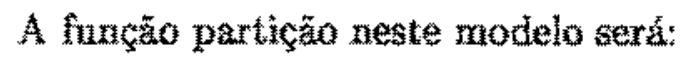

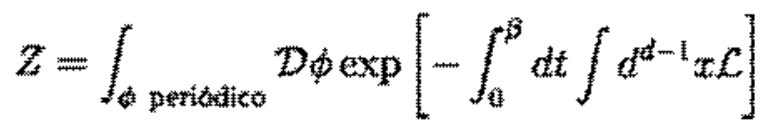

onde 2 pode ser divididu ern duas partes, a parte correspondente a partícula livre:

$$
c_{0}=-\frac{1}{2} \partial_{n} \phi^{\mu} \phi
$$

e a parte de interacäa:

$$
C_{1}=\frac{1}{6} \lambda \phi^{3}
$$


Podemos deduzir as regras de Feynman a temperatura finita inserindo fontes externas na lagrangeana, obtendo a função partiçāo da forma:

$$
Z=\int \mathcal{D} \phi \exp \left\{\int_{0}^{\beta} d \tau \int d^{d-1} x\left[\mathcal{L}_{1}(\phi)+\frac{1}{2}\left(\phi \nabla^{2} \phi+\phi \partial_{\tau}^{2} \phi\right)+\eta \phi\right]\right\}
$$

Podemos assim extrair a parte de interação da integral funcional e, fazendo uma transformada de Fourier nas cordenadas espaciais e usando uma série de Fourier nas coordenadas ternporais, obtemos:

$$
Z(\eta)=\exp \left[-T \sum_{k_{0}=2 \pi i n T} \int \frac{d^{d-1} \vec{k}}{(2 \pi)^{d-1}} S_{\mathrm{I}}\left(\frac{-\delta}{\delta \eta}\right)\right] Z_{0}(\eta)
$$

onde $\vec{k}$ são as componentes espaciais do momento e $Z_{0}(\eta)$ é a funçāo partição de uma partícula livre com fontes externas, isto é:

$$
\begin{aligned}
Z_{0}(\eta) & =\int \mathcal{D} \phi \exp \left\{-T \sum_{n} \int \frac{d^{d-1} \vec{k}}{(2 \pi)^{d-1}}\left[\frac{1}{2} \phi k^{2} \phi+\eta \phi\right]\right\} \\
& =\exp \left\{T \sum_{n} \int \frac{d^{d-1} \vec{k}}{(2 \pi)^{d-1}}\left[\frac{1}{2} \frac{\eta\left(k_{0}, \vec{k}\right)^{2}}{k^{2}}\right]\right\}
\end{aligned}
$$

Assim vemos claramente que as regras de Feynman a temperatura finita são as mesmas que a temperatura zero com as mudanças:

$$
\int \frac{d^{d} k}{(2 \pi)^{d}} \rightarrow i T \sum_{n} \int \frac{d^{d-1} \vec{k}}{(2 \pi)^{d-1}}
$$

para cada loop interno, onde:

$$
k_{0}=2 \pi i n T
$$

Como não usamos a forma explícita de $\mathcal{L}_{1}$, estas regras valem para qualquer teoria bosônica, inclusive a QCD sem fermions, ou bósons sob ação de um campo gravitacional.

Para entender o mecanismo da teoria vamos calcular a função de 2 pontos da teoria escalar em 6 dimensōes na ordem de um loop representada pelo gráfico: 


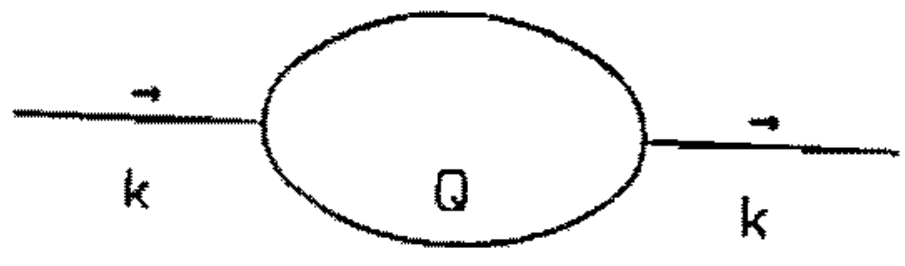

Usando as regras de Feynman obtemos:

$$
\Pi(p)=\lambda i T \sum_{k_{0}=2 \pi i n T} \int \frac{d^{5} \vec{k}}{(2 \pi)^{5}} \frac{1}{k^{2}} \frac{1}{(k-p)^{2}}
$$

A primeira dificuldade é calcular o somatôrio sobre $k_{0}[10]$. Para isso, podemos utilizar a funçäo ctgh( $\pi$ ) que tem polos simples com residuo +1 em todos os pontos $z=$ in onde $z$ é urn número inteiro, como é mostrado na Gigura:

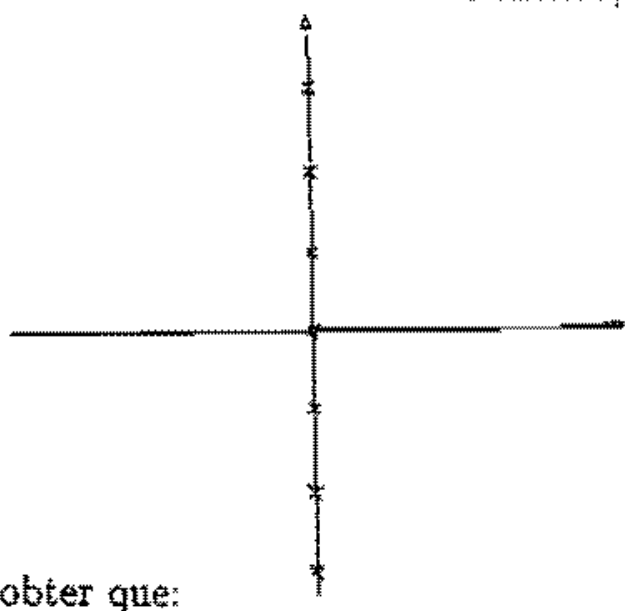

Assim, podernos obter que:

$$
\sum_{R=-\infty}^{\infty} f\left(k_{0}=2 \pi i n T\right)=\frac{1}{2 \pi i T} \int_{c} \frac{d z}{2} f(z) \operatorname{ctgh}\left(\frac{z}{2 T}\right)
$$

onde $f(z)$ é qualquer função que näo tenha polos no interior do contorno $C$ que errolve o eixo imaginário representado pela figura: 


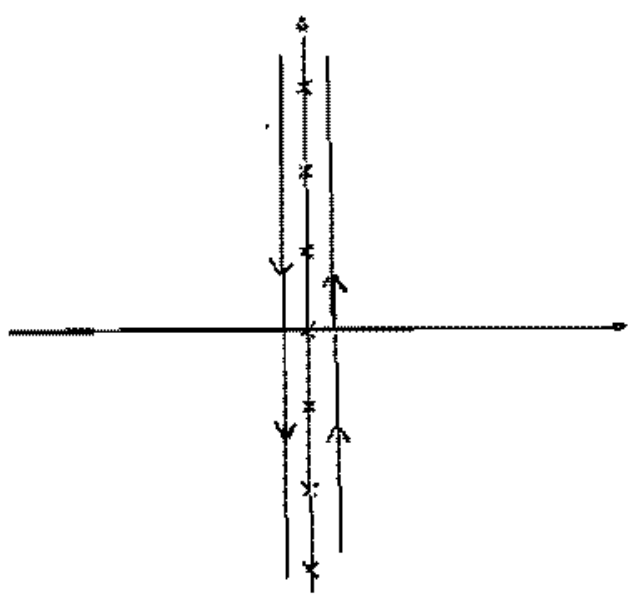

Este contorno pode ser representado por duas retas, uma que vai de $-i \infty+\varepsilon$ a $i \infty+\varepsilon$ e outra que vai de $i \infty-\varepsilon$ a $-i \infty-\varepsilon$, onde $\varepsilon$ é arbitrariamente pequeno, e $f(z)$ näo tem polos no eixo imaginário. Podemos fazer uma transfornação $z \rightarrow-z$ na segunda reta deste contorno: obtendo:

$$
\begin{aligned}
T \sum_{k_{0}} f\left(k_{0}\right)= & \frac{1}{2 \pi i} \int_{-i \infty+\infty+\varepsilon}^{i \infty \infty+\varepsilon} d z \frac{1}{2}[f(z)+f(-z)] \operatorname{ctgh}\left(\frac{z}{2 T}\right) \\
= & \frac{1}{2 \pi i} \int_{-i \infty}^{i \infty} d z \frac{1}{2}[f(z)+f(-z)] \\
& +\frac{1}{2 \pi i} \int_{-i \infty+\infty}^{i \infty \infty+c} d z\left[f(z)+f(-z) \mid N\left(\frac{z}{T}\right)\right.
\end{aligned}
$$

onde $N(x)$ é a funçăo distribuiçăo de Bose:

$$
N(x)=\frac{1}{e^{x}-1}
$$

Quando substituinos (1.22) na funçäo de 2 pontos da teoria escalar (1.19) é fácil ver que o primeiro terno de (1.22) nada mats é que o propagador a temperatura zero da teoria euclidianizada, portanto todas as contribuiçōes térmicas são obtidas apenas do segundo termo. O segundo termo pode ser calculato fechando-se o contorno dessta torma: 


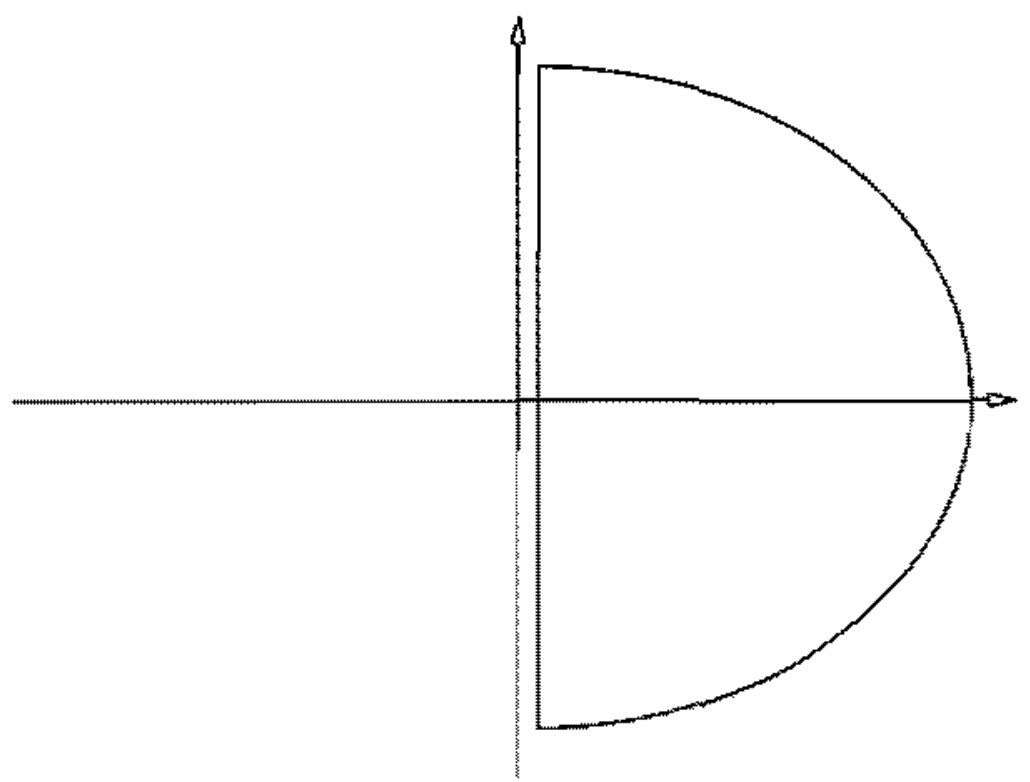

onde $R \rightarrow \infty$

O resultado desta integral é o valor dos residuos de $f(z)$ e $f(-z)$ no fnterior deste contomo. Da fómula (1.19) veroog que os únicas residuos provém dos polos dos propagadores das particulas interuas, quando estas esta na "concha" de massa. lsto corresponde a "quebrar" os propagadores nos loops, transformando os graficos com loops em graficos de espalhamento por particulas térmicas na "concha" de massa. Desta forma, obternos para (1.19) a formula:

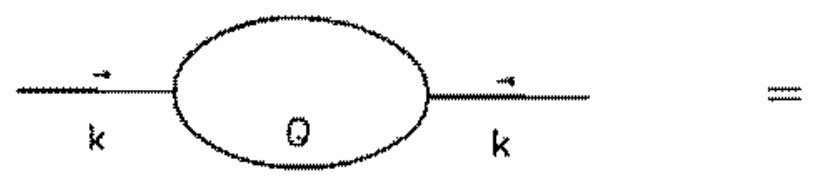

$$
=\int \frac{d^{5} \vec{k}}{|\vec{k}|} N\left(\frac{|\vec{k}|}{T}\right)-\left.\int_{0}\right|_{0} ^{k}=\frac{\mid k}{0} \div(k \leftrightarrow-k)
$$

Este método é analogo para qualquer teoria de campos. Desta forma, 
podemos sempre calcular gráficos de Feynman a temperatura finita como espalhamentos de partículas térmicas.

A função de dois pontos da teoria escalar, usando este método, será:

$$
\Pi(p)=-\lambda^{2} \int \frac{d^{5} k}{(2 \pi)^{5}} \frac{1}{2 k} N\left(\frac{k}{T}\right)\left[\frac{1}{p^{2}+2 k \cdot p}+k \leftrightarrow-k\right] .
$$

Em termos de $x \equiv \cos \theta$, onde $\theta$ é o ângulo entre $\mathbf{k}$ e p obtemos:

$$
\Pi(p)=\lambda^{2} \frac{p^{2}}{64 \pi^{3}} \int_{-1}^{1} \frac{1-x^{2}}{\left(p_{0}-|\bar{p}| x\right)^{2}} d x \int_{0}^{\infty} k^{3} N\left(\frac{k}{T}\right) \frac{d k}{k^{2}-\left[\frac{p^{2}}{2\left(p_{0}-|\bar{p}| x\right)}\right]^{2}}
$$

A partir deste ponto podemos fazer uma expançāo para achar os termos dominantes na temperatura ou podemos resolver esta integral exatamente por um método que será explicado mais adiante. Vamos considerar primeiramente o método usual de expandir em termos dominantes em altas tempereturas. Para isto observamos que os momentos das partículas internas nos loops é de ordem $T$ quando $T>p$. Assim podemos expandir o denominador da integral radial de (1.26) em potências da forma:

$$
\frac{k^{2}}{k^{2}-\left[\frac{p^{2}}{2\left(p_{0}-|\vec{p}| x\right)}\right]^{2}}=1+\mathcal{O}\left(\frac{1}{k^{2}}\right)
$$

e usando que[11]:

$$
\int_{0}^{\infty} N\left(\frac{k}{T}\right) k d k=\frac{\pi^{2} T^{2}}{6}
$$

obtemos, para o termo dominante:

$$
\Pi_{d}(p)=\lambda^{2} \frac{T^{2}}{96 \pi}\left(1-\frac{p_{0}^{2}}{|\vec{p}|}\right)\left(1-\frac{p_{0}}{2|\vec{p}|} \ln \left|\frac{p_{0}+|\vec{p}|}{p_{0}-|\vec{p}|}\right|\right)
$$

e os termos sub-dominantes podem ser obtidos de forma análoga.

A partir da equaçāo (1.26), porém, podemos obter a função de dois pontos de forma fechada, isto é, podemos obter $\Pi(p)$ como uma função de $p$ que envolve todos os termos, dominantes e sub-dominantes na temperatura. Este 
método será utilizado posteriormente para o cálculo da função de dois pontos do grấviton e, por isso, será considerado aqui. Para isto usamos a fórmula:

$$
J(y)=\int_{0}^{\infty} N\left(\frac{k}{T}\right) \frac{k d k}{k^{2}-(2 \pi T y)^{2}}=\ln (y)-\frac{1}{2 y}-\psi(y)
$$

onde $y \equiv \frac{i}{4 \pi T} \frac{k^{2}}{p_{0}-p \vec{p} x}$ e $\psi(y) \equiv \frac{d}{d y} l n \Gamma(y)$ é a funçäo psi de Euler. É importante notar que para obtermos o limite de tempo real da funçäo de Green deveruos fazer uma continuação analítica de nosso resultado com tempo irnaginário atravez de uma prescrição específica. Esta prescriçãa, porérn, vai înfluir apenas na parte imaginária da função de dois pontos, que nầo nos interessa neste trabalho.

Utiiizando a função $J$ definida na fórmula (1.30), podemos wotar que a função de dois pontos pode ser escrita como:

$$
\mathrm{\Pi}(p)=\mathrm{n}_{d}(p)-\lambda^{2} \frac{x^{2} p^{2}}{32 \pi} \int_{-1}^{1} \frac{d x\left(1-x^{2}\right)}{\left(p_{0}-\mid \vec{p}\right)^{2}} y^{2} J(y)
$$

onde $\Pi_{d}(p)$ é o termo dominante em altas temperaturas dado pela equação (1.29). O segundo termo desta equação envolve apenas integrais da forma:

$$
I_{n}(q)=\int_{C}^{q} y^{n} J(y) d y
$$

com $n=0,1$ ou 2. Estas integrais podem ser expressas em termos das funções zeta de Ríeman generalizadas e psi de Euler, além de funções elementares. Estas integrais estäo computadas no apendice A. Desta forma, definindo:

$$
q\left(p_{0}\right) \equiv i \frac{p_{0}+|\vec{p}|}{4 \pi T}
$$

a funçäo de dois pontos nestat teoria pode ser representada como:

$$
\Pi(p)=\Pi_{d}(p)+F\left[q\left(p_{0}\right)\right]-F\left[-q\left(-p_{0}\right)\right]
$$

onde:

$$
F(q)=\frac{k^{2}}{8 \mid \overline{p p^{2}}}\left[\frac{m p^{2}}{16 \pi^{2}} I_{0}(q)-\frac{T^{2} p_{0}}{2 \pi} l_{1}(q)-h^{3} l_{2}(q)\right]
$$

Este resultado fornece uma forma explicita da função de dois pontos desta teoria. Resultado análogo pode ser obtido na teoria de Yang-Mills[12]. 


\section{Capítulo 2}

\section{Divergências Infravermelhas em teorias de campo a temperatura finita}

Eem conhecido que os problemas de divergências infravermelhas săo muito mais severos em uma teorita que contem acoplamentos entre partículas sem massa, como a QCD, do que numa teoria como a eletrodinâmica quântica. É conhecido que en temperatura finita as divergências infravermelhas tornam irapossivel descrever a transiçäo de fase de segunda ordem da teoria escalar com acoplamento $\lambda \phi^{4}$ próxima da tempertura crítica $T_{c}$ pois, neste caso, os bósons da teoria deixam de ser massivos. Na teoria de Yang-Mills essas divergências säo ainda mais severas, tornando imposivel descrever a função partiçäo além da quinta ordem de perturbação a qualquer temperatura[13].

No caso da teoria $\lambda \phi^{3}$ estas divergências também ocorrem. A temperatura finita, porếm, podemos retirar as divergências desta teoria. Para entender este ponto considere una forma geral de uma integral sobre um loop de um grafico qualquer que contribua para a funçẵo partição:

$$
\cong T \sum_{k_{0}} \int u \vec{k} \mathcal{D}\left(k_{0}, \vec{k}\right) \times \mathcal{D}\left(k_{0}+p_{10}, \vec{k}+\vec{p}_{1}\right) \times \mathcal{D}\left(k_{0}+p_{20}, \vec{k}+\vec{p}_{2}\right) \times \cdots
$$

onde $\mathcal{D}$ é o propagador,

As divergências infravermelhas surgern apenas quando $k_{0}=0$ no primeiro propagador (ou $h_{0}+p_{i 0}=0$ nos outros). Além disto, ela vem da regiào 
$\vec{k} \rightarrow 0$ no primeiro propagador (ou $\vec{k}+\vec{p}_{i} \rightarrow 0$ ). Em suma, as divergências infravermelhas vem do fato de:

$$
\mathcal{D}\left(k_{0}=0, \vec{k} \rightarrow 0\right) \rightarrow \infty
$$

Porém quando calculamos a função de dois pontos da teoria $\lambda \phi^{3}$ em primeira ordem de perturbação, obtivemos que o termo dominante em altas teroperaturas era:

$$
\Pi_{d}(p)=\lambda^{2} \frac{T^{2}}{96 \pi}\left(1-\frac{p_{0}^{2}}{|\vec{p}|}\right)\left(1-\frac{k_{0}}{2|\vec{p}|} l n\left|\frac{p_{0}+|\vec{p}|}{p_{0}-|\vec{p}|}\right|\right)
$$

e assim podemos observar que:

$$
m_{t}^{2} \equiv \Pi\left(p_{0}=0, \bar{p} \rightarrow 0\right)=\lambda^{2} \frac{T^{2}}{96 \pi}
$$

Desta formula observamos que $m_{t}$ representa uma massa térmica que aparece naturalmente da teoria. Por outro lado, o propagador efetivo pode ser obtido por sucessivas inserçōes da funçāo de dois pontos conforme a figura:
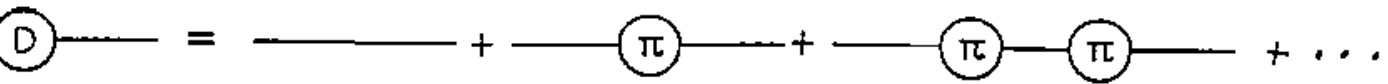

Desta forma, podemos utilizar que:

$$
\mathcal{D}(p)=\frac{1}{p^{2}} \div \frac{1}{p^{2}} \Pi(p) \mathcal{D}(p)
$$

onde $\mathcal{D}$ é o propagador desta teoria, obtendo:

$$
\mathcal{D}_{e f f}(p)=\frac{1}{p^{2}-\Pi(p)}
$$


Este propagador fetivo apresenta naturalmente uma massa térmica $m_{t}$ e, assim, näo diverge quando $\eta_{0}=\hat{0}_{i} \vec{p} \rightarrow 0$. Este propagador pode então ser utilizado para se calcular as ordens maiores na teoria de perturbaçón sem termos problemas de divergências infravermelhas.

Na teoria de Yang-Mills, infelizmente, näo é isso que acontece. Vamos analisar a estrutura do tensor de polarizaçăo de um gloon. Em temperatura zero, devido a simetria de Lorenz da teoria, este tensor só pode depender de dois tensores covariantes simétricos, desta forma:

$$
\Pi_{\mu \nu}=a\left(p^{2}\right) g_{\mu p}+b\left(p^{2}\right) \frac{p_{\mu} p_{\nu}}{p^{2}}
$$

e, devido a transveraalidade (identidade de Ward no gauge de Feynman):

$$
p^{3} \Pi_{n=0}=0
$$

este tensor tem a forma bem conhecida de termperatura zero:

$$
\Pi_{\mu \nu}=F\left(p^{2}\right)\left(g_{\mu \nu}-\frac{p_{\mu} p_{\nu}}{p^{2}}\right)
$$

A temperatura finita, porém, nö̆o temos mais a simetria de Lorenz, pois passamos a ter un referencial privilegiado, o referencial de repouso do plasmat Deste modo, alem do quadri-vetor momento $p_{p,}$, temos também o quadrivetor velocidade do plasma $u_{35}$ que no referencial de repouso do plasman sera ${ }_{i j}=(1,0) .0$ tensor de polarizaçäo tera entă a forma geral:

$$
\Pi_{\mu \nu}=a\left(p^{2}\right) g_{j \nu}+b\left(p^{2}\right) \frac{p_{\mu} p_{\nu}}{p^{2}}+c\left(p^{2}\right) u_{\mu} u_{y}+d\left(p^{2}\right) \frac{u_{\mu} p_{\nu}+p_{u} w_{\nu}}{p_{0}}
$$

que, novamente, pela transversalidade (2.8) pode ser reduzido a:

$$
\Pi_{\mu \nu}=F\left(p^{2}\right) A_{\mu \nu}+G\left(p^{2}\right) B_{\mu \nu}
$$

onde os tensores $A$ e $B$ são:

$$
\begin{aligned}
& A_{00}=A_{0 j}=A_{j 0}=0 \\
& A_{i j}=o_{i j}-\frac{p_{i j} p_{i}}{|p|^{2}}
\end{aligned}
$$

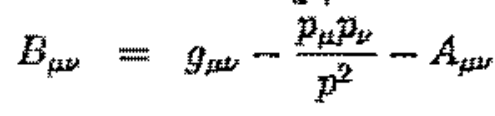


E, igualmente para o propagador, teremos que:

$$
\mathcal{D}_{\mu \nu}=\frac{g_{\mu \nu}}{p^{2}}+M\left(p^{2}\right) A_{\mu \nu}+E\left(p^{2}\right) B_{\mu \nu}
$$

Os tensores $A$ e $B$ correspondem a modos de vibraçāo do plasma. O tensor $A$ corresponde à componente espacial $\Pi_{i j}$ do tensor de polarização, enquanto o tensor $B$ corresponde às componentes temporais $\Pi_{0 \mu}$. Desta forma, podemos podemos ver que o modo $B$ é reponsavel pela propagação da componente $G_{0 i}$ do campo cromodinâmico, e, por analogia com o eletromagnetismo, dizemos que este é o modo cromoelétrico da teoria. Da mesma forma, o modo $A$ é a parte cromomagnética.

Vamos agora calcular o termo dominante em altas temperaturas do tensor de polarização de uma teoria de Yang-Mills. As regras de Feynman desta teoria sāo dadas no apêndice B. Em ordem de $g^{2}$, no gauge de Feynman, a parte dependente da temperatura do tensor de polarizaçāo será:

$$
\begin{gathered}
\Pi_{\mu \nu}^{a b}(p)=-\frac{g^{2} N \delta^{a b}}{(2 \pi)^{3}} \int \frac{d^{3} k}{2 k} N\left(\frac{k}{T}\right) \times \\
\times\left[\frac{\left(2 p^{2}-4 p \cdot k\right) g_{\mu \nu}+8 k_{\mu} k_{\nu}+4\left(k_{\mu} p_{\nu}+k_{\nu} p_{\mu}\right)-2 p_{\mu} p_{\nu}}{p^{2}+2 k \cdot p}+(k \leftrightarrow-k)\right]
\end{gathered}
$$

A partir desta equação, podemos observar que os termos dominantes erm altas temperaturas, isto é, os termos que envolvem maiores potências do momento interno $k$ serão dados por:

$$
\Pi_{d \mu \nu}^{a b}(p)=\frac{N \delta^{a b}}{4 \pi^{3}} \int \frac{d^{\beta} k}{k} N\left(\frac{k}{T}\right)\left(\frac{p^{2} k_{\mu} k_{\nu}}{(k \cdot p)^{2}}-\frac{k_{\mu} p_{\nu}+k_{\nu} p_{\mu}}{k \cdot p}+g_{\mu \nu}\right)
$$

Quando $p_{0}=0$ podemos observar que a parte espacial $\Pi_{d i j}$ se anula quando fazemos as integrais angulares, obtendo assim que:

$$
\Pi_{d \mu \nu}^{a b}\left(p_{0}=0\right)=N g^{2} \delta^{a b} \frac{T^{2}}{3}
$$

ou, usando a notação da equaçāo (2.11) temos:

$$
F_{d}=0 \quad ; \quad G_{d}=N g^{2} \frac{T^{2}}{3} B_{\mu \nu}
$$


$O$ valor de $G_{d}$ é chamado de massa elétrica do gluon. Isto permite uma ressomagem da parte elétrica do propagador (os termos do propagador proporcionais a $B_{\mu \nu}$ ) analoga to que acontece na teoria escalar. Usando-se que:

$$
\mathcal{D}_{\mu \nu}=\frac{g_{\mu \nu}}{p^{2}}+\frac{1}{p^{2}} \mathcal{D}_{\mu \rho} \Pi_{\psi}^{p}
$$

vernos que a parte elétrica do propagador do gluon näo irt contribuir para divergềcias infravermelhas, porém quando fazemos o mesmo para a parte raaguética (proportionais a $A_{p a v}$ ) o mesmo näo acontece.

A princípio parece que se pudessemos encontrar uma massa magnética em ordem superior na teoria de perturbaçäo este problema estaria resolvido, porérn analisernos o que aconteceria apenas nos diagramas de ordem $g^{2 N}$ contendo $N$ vértices quárticos de glvons "magnéticos", com una massa $m_{m}(T)$. Esta contribuição será da forma:

$$
Z(T) \sim(2 \pi T)^{N+1} g^{2 N} \int d^{3} \ddot{p}_{1} \cdots d^{3} \vec{p}_{N+1} \sum_{p o s} \prod_{i=1}^{2 N}\left[q_{0 i}^{2}+\vec{q}^{2}+m_{m}(T)^{2}\right]^{-1}
$$

onde $q_{i}$ nada mais é que urna combinaçăa linear de $p_{i}$, e podemos assim podemos trocar nas integrais e sornatórios $p_{i}$ por $q_{i}$. Novamente a parte mais divergente corresponde a $q_{i 0}$, assima podemos escrever:

$$
\begin{aligned}
Z(T) & \sim(2 \pi T)^{N+1} g^{2 N} \int d^{3} \vec{p}_{1} \cdots d^{3} \vec{p}_{N+1} \prod_{i=1}^{2 N}\left[\vec{q}^{2}+m_{m}(T)^{2}\right]^{-1} \\
& \sim g^{6} T^{4}\left(\frac{g^{z} T}{m(T)}\right)^{N-3}
\end{aligned}
$$

Note que a divergência ultravioleta que aparece em $(2.20)$ é apenas aparente, pois essas divergênciass não aparecern se considerarmos todos os termos do soratónios, pois como vimos sempre aparece um termo do tipo $N(|\vec{p} ;| T)$ que cancela todas as divergências ultravioleta que aparecerem. Na fórmula (2.21) percebemos que se tivermos $m(T)<g^{2} T$ os termos de ordens maiores em $N$ seräo maiores que os de orden menor, impossibilitando assim a teoria de perturbaçōes. O termo de ordern $g^{2} T$ pode aparecer devido a correçōes de ordem superiores em $\Pi_{\text {mag }}$, mas por um raciocinio semelhante ao apresentado é facil ver que todas as ordens contribuiräo, isto é, em todas as ordens terenos que:

$$
\Pi_{\text {raa }}\left(k_{0,} \vec{k} \rightarrow 0\right) \sim g^{2} T
$$


Devido a isso a funçăo partição em QCD năo pode ser calculado perturbativamente além da quinta ordem na constante de acoplaraento. Por todos estes motivos, só podemos tentar entender como pode ocorrer o cancelamento de divergências infravermelhas se usamos métodos nato perturbativos. 


\section{Capítulo 3}

\section{Estudo do comportamento infravermelho na aproximação "ladder"}

Como vimos, os estudo da teoria de Yang-Mills a temperatura finita năo pode ser feito perturbativamente alén da quinta ordem na constantede acoplamento. Para tentar entender o cancelamento das divergencias infravernelhas nesta teoria necessitamos de alguma forma de tratamento não perturbativo. Para isto usamos as equaçōes de Schwinger-Dyson. É bern conhecido que, em QCD, estas formam um conjunto infinito de equaçôes, pois a equação para a função de $n$ pontos depende das funçōes de $n+1$ en $n+2$ pontos, 0 que torna o problema extremamente complicado. Para contornar este problema, terncs de truncar de alguma foma estas equaçoes. Neste trabalho usamos a aproximação "Tadder", como sera explicado mais tarde. Esta aproximaçäo foi utilizada por Mandelstam para entender um mecanismo pelo qual o quark pode ser confinado a ternperatura zero, foi utilizado tambến por Jackiw e Templeton para mostrar como teorias super-renormalizaveis curam as divergencincias infravenmelhas.

Vamos estudar a princípio a teoria escalar com acoplamento $\lambda \phi^{3}$ en 6 dimensōes a temperatura $T$. Como vimos no capítulo anterior esta teoria năo sofre problernas com divergências inifravermelhas se fizermos uma resomagem no propagador, mas usaremos aquĩ o mesmo método que usaremos posteriomente em QCD. Para isso considere as equaçoes de Schwinger-1yson truncadas da forma: 


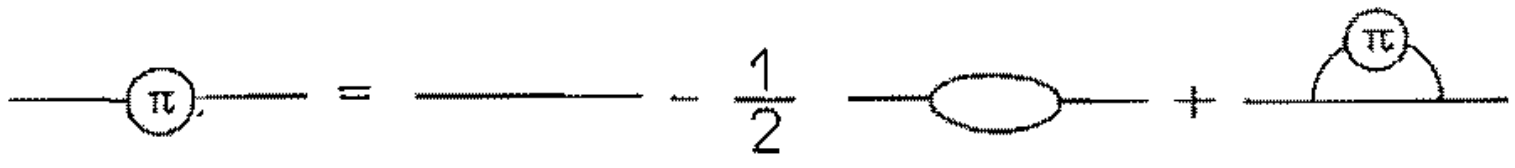

Note que para os diagramas terern o mesmo fator combinatorial $\frac{1}{2} \mathrm{em}$ todas as ordens, necessitarnos do diagrama de urn loop. Para deduzirmos a fómula da pressẫo dada pelo gráfico:

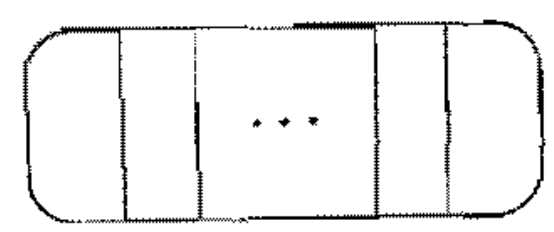

usamos a fórmula:

$$
\left(\frac{\delta P}{\delta \mathrm{D}_{0}}\right)_{1 \mathrm{PI}}=-\frac{T}{2} \tilde{P}=-\frac{T}{2}\left(\mathrm{\Gamma}(p)+p^{2}\right)
$$

onde $D_{\text {te }}$ o propagador da partícula livre e IPI indica que somente gráficos irreduciveis por uma partícula contribuern. Pelo gráfico (3.1) venos que (3.3)' implica ex:

$$
P=P_{0}-\frac{T}{2} \frac{1}{4} \sum_{p_{0}} \int \frac{d^{\tilde{j}} \vec{p}}{(2 \pi)^{5}} \frac{1}{\tilde{p}^{2}+p_{0}^{2}}\left[\tilde{p}\left(p_{0}, \tilde{p}\right)+\frac{1}{3} \tilde{p}^{(1)}\left(p_{0}, \tilde{p}\right)\right]
$$


O fator $\frac{1}{4}$ é devido a que há quatro forma de cortax o diagrama (3.2) obtendo $\bar{P}$ na aproximaçäo ladder. A única excessāo ocorre na ordern mais baixa onde aparece of fator $\frac{1}{3}$. A fórmula $(3.4)$ pode ser representada graficamente da forma:

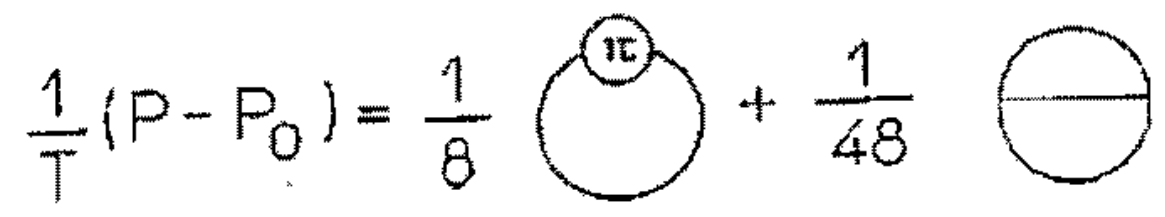

A soma sobre $p_{0}$ é feitas sobre todas as frecuências $p_{0}=2 \pi n T$, porém somente os modos com $p_{0}=0$ coniribuem para as divergenncias infravermelhas. Quando calculamos esses modos surge uma divergência ultravioleta aparente, corno apareceu no final do capítulo anterior, mas, como vimos, esta divergência ê meramente aparente, pois se sornássemos sobre todos os modos $n$ apareceria naturalmente un fator de Bose do tipo:

$$
N(k)=\frac{1}{\exp \left(\frac{k}{2}\right)-1}
$$

cancelando essas divergèncias. O fator de Bose gera, de certa forma, uxn cutoff ultravioleta de ordem $T$ nas integrais sobre os momentos, que usaremos aqui. Para entender o cancelamento das divergências infravermelhas varnos colocar um um cut-off $m$ na região do infravermelho. O cancelamento das divergências infravermelhas implicam que podemos fazer $m \rightarrow 0$. Desta forma podemos escrever ( 3.4$)$ da forma:

$$
P=P_{0}-\frac{T}{8} \int_{m}^{T} \frac{d^{5} p}{(2 \pi)^{5}} \frac{1}{p^{2}} P\left(p_{0}=0, p\right)+\cdots
$$

onde as reticências se referens a termos de ordem inferior e convergentes na regiảo do infravermelho. 
Usando as equaçoes de Schwinger-Dyson da forma (3.1) obtemos para a função de dois pontos $\widetilde{P}\left(p_{0}=0, p\right)$ a equacäo integral da forma:

$$
\widetilde{p}(p)=\Pi(p)+p^{2}=-\frac{\lambda^{2} T}{(2 \pi)^{5}} \int_{m}^{T} \frac{d^{5} k}{(p+k)^{2}}\left[\frac{1}{2 k^{2}}+\frac{\Pi(k)}{k^{2}}\right]
$$

Esta equaçäo é ruuito complicada de se resolver exatamente, por isto vamos nos restringir à parte responsável pela divergência infravermelhà. Isto é possivel se usarmos um procedimento de rnaximização do tipo:

$$
(p+k)_{\max }^{2}= \begin{cases}p^{2} & \text { se } p>k \\ k^{2} & \text { se } k>p\end{cases}
$$

Usando isto, da equacão (3.8) obtemos:

$$
\Pi_{\max }(p)=-p^{2}+\frac{\lambda^{2} T}{12 \pi^{3}}\left[\frac{p}{3}-\frac{T}{2}+\frac{1}{6} \frac{m^{3}}{p^{2}}-\frac{1}{p^{2}} \int_{r n}^{p} d k \Pi_{\operatorname{rmax}}(k)-\int_{p}^{T} \frac{d k}{k^{2}} \Pi_{\max }(k)\right]
$$

Comparando este resultado com a equaçäo original (3.8), vernos que a primeira iteração de (3.10) com $m \rightarrow 0$ é:

$$
\Pi_{\max }^{(1)}=-p^{2}+\frac{\lambda^{2} T}{24 \pi^{3}}\left[1-\frac{2}{3} \frac{p}{T}\right]
$$

que concorada completamente com (3.8) no limite de altas temperaturas. $\mathrm{Na}$ próxima ordern, este procedimento leva a resultados que concordam satisfatoriamente com (3.8), que se torma cada vez mais complicado dese manejar. Como estamos interessados no comportamento das divergencieias infraverneIhas em altas temperaturas, será suficiente para nossos objetivos nos restringirmos a estudo da equação (3.10). A partir deste ponto varnos chamar $\Pi_{\text {rnax }}$ de II por simplicidade. Após sucessivas iteraçōes encontramos em (3.8) divergências infravermelhas na série perturbativa de II:

$$
\begin{aligned}
\Pi(p)=-p^{2}+\frac{\alpha}{2} T^{2}- & \alpha^{2} T^{2}\left(\frac{T}{p}\right)+\alpha^{3} T^{2}\left(\frac{T}{p}\right)^{2} \ln \left(\frac{p}{m}\right)-\frac{7}{6} \alpha^{4} T^{2}\left(\frac{T}{p}\right)^{3} \frac{p}{m} \\
& +\frac{19}{36} \alpha^{5} T^{2}\left(\frac{T}{p}\right)^{4}\left(\frac{p}{m}\right)^{2}-\frac{149}{340} \alpha^{6} r^{2}\left(\frac{T}{p}\right)^{5}\left(\frac{p}{m}\right)^{3}+\cdots
\end{aligned}
$$


onde $\alpha=\frac{\lambda^{2}}{12 \pi}$. Esta fórmula, combinada com a equação (3.7), exibe as divergencias infravermelhus da pressão $(m \rightarrow 0)$ dadas por:

$$
P_{\text {aiv }}=-\frac{T^{6}}{96 m^{3}}\left[\alpha^{3} \ln \left(\frac{T}{m}\right)-\frac{7}{6} \alpha^{4}\left(\frac{T}{m}\right)+\frac{16}{36} \alpha^{5}\left(\frac{T}{m}\right)^{2}-\frac{149}{540} \alpha^{6}\left(\frac{T}{m}\right)^{3}+\cdots\right]
$$

Vale notar que estas sấ tem a mesma estrutura das divergências que aparecem exn QCD. Apesar de que a equaçä́ (3.10) nāo pode ser resolvida perturbativamente, vamos mostrar que existe uma soluçäo näo perturbativa finita $\mathrm{em} m \rightarrow 0$. Para ssto wamos definir;

$$
x=\frac{\alpha T}{p} ; f(x)=\frac{\Pi}{p^{2}}
$$

Asscin a equaçăo (3.10) se toxna (eliminando-se o termo não homogêneo $\left.m^{3}\right)$ :

$$
f(x)=-1+\frac{x}{3}-\frac{x^{2}}{2 \alpha}-x^{2} \int_{a}^{x} \frac{d y}{y^{2}} f(y)-x^{4} \int_{x}^{\frac{a T}{m}} \frac{d y}{y^{4}} f(y)
$$

Nesta fórmula a teoria de peruurbaçóes corresponde a resolver esta equação por série de potencias ern $x$ que, como vimos, ê divergente na regiấ do infravermelho. Esta equaçăo, porém, pode ser transformada nesta equação diferencial:

$$
x^{2} f^{n}-5 x f^{\prime}+(8-2 x) y=x-8
$$

Esta equaçäo diferencial pode ser resolvida usndo-se as funçōes de Bessel modificadas. Para a parte homogênea temos a soluçâa:

$$
f(x)=A x^{3} L_{2}(\sqrt{8 x})+B x^{3} K_{2}(\sqrt{8 x})
$$

Usando métodas usuais de equaçóe diterenciais podemos acha uma soluçăo particular da equacảo não homogenea, e assim a soluçăo geral:

$$
\begin{aligned}
f(x)= & {\left[A+2 \int_{z}^{\frac{x^{x}}{m}} d t\left(\frac{8}{t^{4}}-\frac{1}{t^{3}}\right) K_{2}(\sqrt{8 t})\right] x^{3} I_{2}(\sqrt{8 x}) } \\
& +\left[B+2 \int_{z}^{\frac{\mathrm{eT}^{m}}{m}} d t\left(\frac{8}{t^{4}}-\frac{1}{t^{3}}\right) I_{2}(\sqrt{8 t})\right] x^{3} K_{2}(\sqrt{8 x})
\end{aligned}
$$


As constantes $A$ e $B$ podem ser fixadas em termos dos parâmetros $\frac{a T}{m}$ e $\alpha$ substituindo-se (3.18) em (3.15). Cono a funçäo $I_{2}(x)$ cresce exponencialmente com $x$, nós teremos uma divergência quando $m \rightarrow 0$, a menos que $A=0$. Na realidade, podemos achar uma solução consistente com a equação integral impondo que $A$ se anula enquanto $B$ vale:

$$
B=\frac{1}{K_{3}(\sqrt{8 \alpha})}\left[2 I_{3}(\sqrt{8 \alpha}) \int_{\alpha}^{\infty} d t\left(\frac{8}{t^{4}}-\frac{1}{t^{3}}\right) K_{2}(\sqrt{8 t})-\sqrt{\frac{8}{\alpha^{7}}}\right]
$$

Como a funçäo $K_{2}(x)$ decresce exponencialmente para grandes valores de $x$, é possivel observar que $f(x)$ é ura funçăo decrescente eno $x$. Como grandes valores de $x$ correspondem a pequenos momentos [cf. ex. (3.14)], este comportamento implica na convergência da integral (3.7) quando $m \rightarrow$ 0. Portanto as divergência infravermelhas da pressão nesta aproximaçä̌ se camcelarn, apesar de aparecerem perturbativrnente.

Vanos considerar agora a teoria de Yang-Mills. Como vimos no capítulo anterior, a parte elétricta do tensor de polarização $\Pi_{00}$ exibe o surgimento de uma massa térmica que elimina as divergéncia infravernelhas devidas a este setor, porérn a parté magnética năo tem esta propriedade. Assinn as divergências desta teoria estäo relacionactas somente com a parte magnética, que, associado a que estas divergências ocorrem somente quando a componente temporal do momento se anula, permite afirmar que o problema está ligado a parte euclidiana tri-dimensional da teoria, que vamos estudar.

Apesar da analise ser mais complicada que na teoria escalar devido aos acoplarnentos quárticos de giúons e a presença de fantasmas, podemos proceder de modo análogo a teoria escalar. Usando uraa versão truncada das equaçōes de Schwinger-Dyson representada graficamente para os glúons por: 

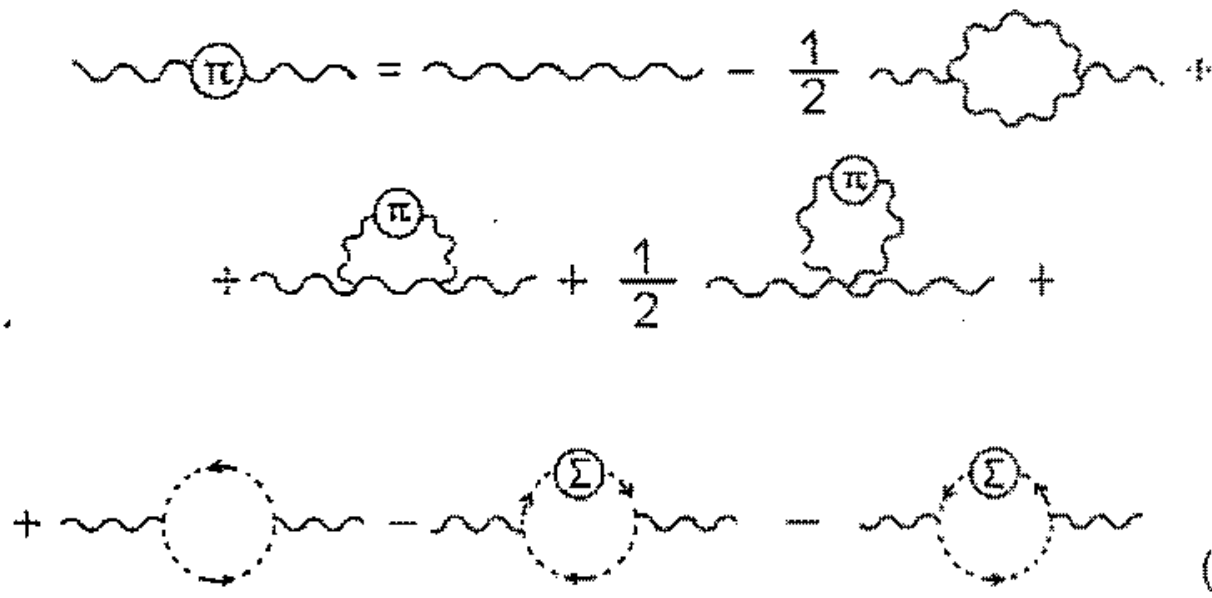

e para os fantasmas por:

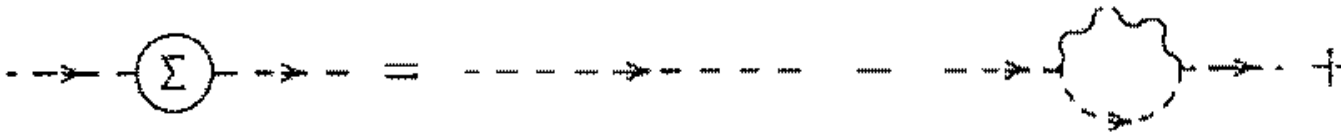

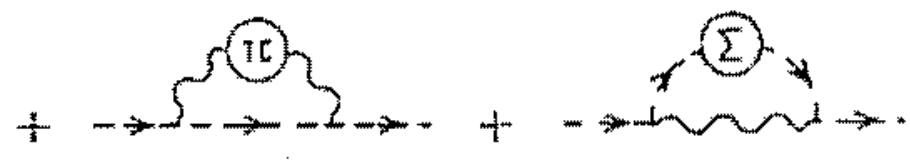

podemos podernos achar uma ecpressäo para pressâo analoga a teoria escalar da forma:

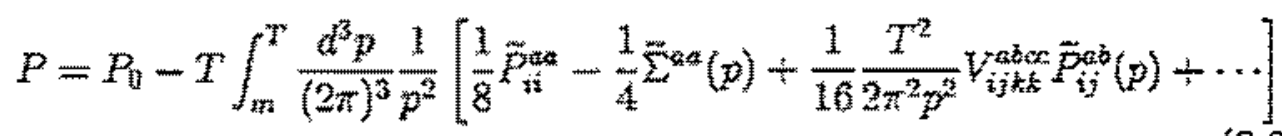

onde $\bar{P}$ e $\bar{\Sigma}$ zão as funços de dois pontos do gluton e dos fantasmas respecivamente, que precishan ser calculadas nesta aproximaçāo, e $V$ representa vértice quártico de glúons näo perturbado. Note que esta formula é muito mais simples do que fómula exata para pressão em uma teoria de YangMills encontrada na literatura[15]. Esta expressäo pode ser representada graficamente pela figura: 


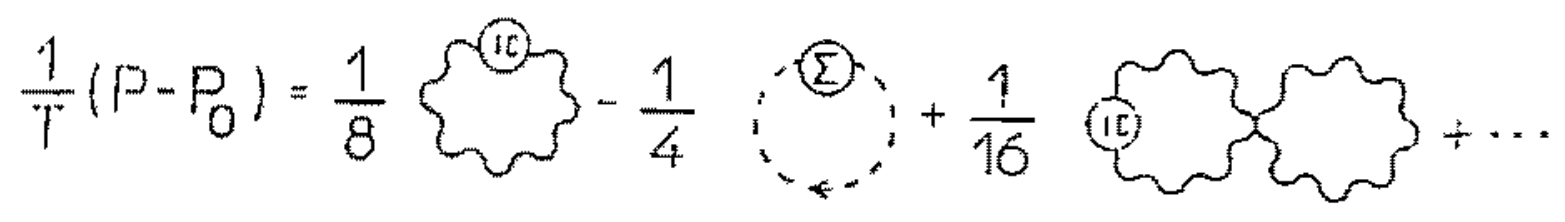

É bem conhecido que qualquer aproximação das equaçōes e SchwingerDyson tropoem grandes vinculos exn urna teoria de gauge, pois as identidades de Ward, em especial a transversalidade, só podem ser obtidas quando consideramos o conjurto completo destas equaçōes. Para contormarmos este problerna adotamos uro procedimento, adotado por Mandelstarn, elirninando os termos longitudinais que surgern das equaçoes de Schwinger-Dyson, que são cancelados pelas contribuiçoes das funçöes de 3 ou mais pontos, que negilgenciamos na aproximaçäo "ladder". Este procedimento é plausível ex como veremos, pernite acharmos urna soluçäo consistente deste conjunto truncado de equaçöes.

Desta forma podemos escrever os gráficos $(3.20)$ e $(3.21)$ para a parte rnagnética do tensor de polarizaçäo:

$$
\vec{p}_{i j}=\left(\hat{d}_{i j}-\frac{p_{i} p_{j}}{p^{2}}\right)\left(\Pi+p^{2}\right)
$$

da seguinte forma:

$$
\begin{aligned}
\Pi(p)= & -p^{2}-\frac{g^{2} N T}{8 \pi^{3}} \int d^{3} k\left[\frac{11}{6} \frac{1}{k^{2}}+\frac{1}{2} \frac{1}{k^{4}} \frac{2\left(2 k^{2}+3 p^{3}\right)(\vec{p} \cdot \vec{k})^{2}-p^{2} k^{2}\left(6 p^{2}+7^{2}\right)}{p^{2}(\vec{p}+\vec{k})^{2}}+\right. \\
& +\frac{1}{3 k^{4}} \Pi(k)-\frac{1}{k^{6}} \frac{5 p^{2} k^{2}-3(\vec{p} \cdot \vec{k})^{2}}{p^{2}(\vec{p}+\vec{k})^{2}}\left(p^{2}+k^{2}\right) \Pi(k)- \\
& \left.-\frac{1}{k^{4}} \frac{p^{2} k^{2}-(\vec{p} \cdot \vec{k})^{2}}{p^{2}(\vec{p}+\bar{k})^{2}} \Sigma(k)\right]
\end{aligned}
$$




$$
\begin{aligned}
\Sigma(p)= & -p^{2}-\frac{p^{2} N T}{8 m^{3}} \int d^{3} k\left[\frac{1}{k^{4}} \frac{(\vec{p} \cdot \vec{k})^{2}}{(\vec{p}+\vec{k})^{2}}-\frac{1}{k^{4}} \frac{(\vec{p} \cdot \vec{k})}{(\vec{p}+\vec{k})^{2}} \Sigma(k)+\right. \\
& \left.+\frac{1}{k^{6}} \frac{p^{2} k^{2}-(\vec{p} \cdot \vec{k})^{2}}{p^{2}(\vec{p}+\vec{k})^{2}} \Pi(k)\right]
\end{aligned}
$$

Na primeira iteração este sistema obtemos a primeira orden de perturbação da funçào de auto energia do glúon e do fantasma. Em ordens superiores na série perturbativa, em conjunto com a fớrmula (3.22), nota-se que aparecem divergências infravermelhas na pressio analogas âs apresentadas na teoria escalar. $\mathrm{E}$ conveniente definirmos as quantidades adimensionais:

$$
\begin{aligned}
& \alpha=\frac{g^{2} N}{3 \pi^{2}} \quad ; \quad z=\frac{\alpha T}{p} \\
& F(x)=\frac{\Pi}{p^{2}} \quad ; \quad G(x)=\frac{\sum}{p^{2}}
\end{aligned}
$$

Analogamente ao tratamento da teoria escalar vamos agora utilizar o procedimento de maximizaçäo ja descrito. Desta forma, após fazermos as integrais angulares obtemos estas quaçöes tipo Volterra:

$$
\begin{aligned}
& F(x)=-1-\frac{13}{12}-H x^{2}+x^{2} \int_{\alpha}^{x} \frac{d y}{y^{2}} G(y)+x^{4} \int_{x}^{\frac{\alpha F}{m}} \frac{d y}{y^{4}} G(y) \\
& g(x)=-\left(1+\frac{\alpha}{2}\right)+x-\int_{\alpha}^{x} d y F(y)-x^{2} \int_{z}^{\frac{\alpha x}{x}} \frac{d y}{y^{2}} F(y)
\end{aligned}
$$

onde

$$
H=\frac{1}{2 \alpha}+\frac{11}{2} \int_{\alpha}^{\frac{\alpha \gamma}{m}} \frac{d y}{y^{2}} G(y)
$$

A teoria de perturbaçöes corresponde a zesolver as equaçón (3.28) e (3.29) en serie de $x$. Por outro lado a regiäo do infravermelho corresponde a região

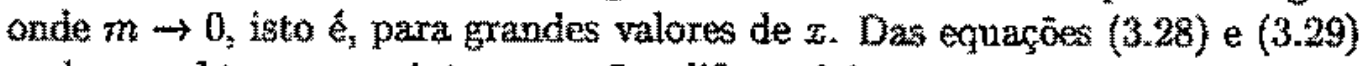
podemos obter as seguintes equaçōes diferenciais:

$$
\begin{aligned}
x^{2} F^{\prime \prime}-5 x F^{\prime}+8 F+2 x G & =-8-\frac{39}{12} x \\
x G^{\prime \prime}-G^{\prime}-2 F & =-1
\end{aligned}
$$


Para obtermos uma solução deste sistema de equaçōes podemos asar a equaçāa (3.31) para escrevermas $G$ em função de $F$ e suas derivadas, e as$\operatorname{sim}$ substituir na equaçāo (3.32) obtendo uma equaçãa diferencial de quarta ordem:

$$
F^{\prime \prime \prime}-\frac{4}{x} F^{\prime \prime \prime}+\frac{12}{x^{2}} F^{\prime \prime}-\frac{24}{x^{3}} F^{\prime}+\left(\frac{24}{x^{4}}+\frac{4}{x^{2}}\right) F=\frac{2}{x^{2}}-\frac{24}{x^{4}}
$$

que descreve o comportamento infravermelho do sistema.

A soluçấo da parte homogểnea desta equação pode ser escrita em termos de funçôe modificadas de Bessel com argumentos complexos. Usando as propriedades destas fumçŏes é possivel mostrar que:

$$
F_{\mathrm{H}}(x)=i x^{2}\left[C_{1} I_{2}\left(\sqrt{8 i x}+C_{2} K_{2}(\sqrt{8 i x})\right]+\right.\text { complexo conjugado }
$$

onde $C_{1}$ e $C_{2}$ são constantes de integraçäa.

Para obtermos uma solução particular da equaçäo (3.33) usamos o método de variaçăo de constantes, usual em equacōes diferenciais. A soluçäo geral é dada por:

$$
\begin{aligned}
F(x)= & i x^{2}\left\{\left[C_{1}+\int_{x}^{\frac{\pi r}{m}} d t\left(\frac{1}{t^{2}}-\frac{12}{t^{4}}\right) K_{2}(\sqrt{8 i t})\right] I_{2}(\sqrt{8 i x})\right. \\
& \left.+\left[C_{2}+\int_{\alpha}^{x} d t\left(\frac{1}{t^{2}}-\frac{12}{t^{4}}\right) I_{2}(\sqrt{8 i t})\right] K_{2}(\sqrt{8 i x})\right\}+c . c .
\end{aligned}
$$

Podemos agora fixar as contantes de integração aplicando esta solução nas equaçöes integrais (3.28) e (3.29). Deste modo obtemos que $C_{1}$ se anula no limite $m \rightarrow 0$ e a constante $C_{2}$ tem ura forma extremamente complicada, porên convergente. Uma vez que $C_{1}$ se anula, das propriedades das funçöes de Bessel podemos ver que $F$ é uma funçäo decrescente em $x$. Uisando estas soluçōes podemos calcular o comportamento de $\Pi$ e $\Sigma$ para pequenos valores de $p$ :

$$
\Pi(p) \cong \frac{1}{2} p^{2} ; \quad \Sigma \cong-\frac{39}{24} p^{2} \quad \text { para } p<<\alpha T
$$

Desta forma a pressâo dada pela fórmula (3.22) converge no infravermelho. Para concluir, reforç̧o que estas conchusöes estäo restritas a aproximação "ladder" , mas processos sernelhantes de cancelamento das divergências infrąverrnelhas devem ocorrer na teoria de Yang-Mills exata. 


\section{Capítulo 4}

\section{Efeitos térmicos em um teoria de campos em espaço curvo}

0 estudo de efétos térmicos de teorias quânticas associadas com a gravitação è um assunto fundamental para entendermos a formação do universo a cosmologia tal a conhecemos nos dias de hoje. A gravitaçâo, ao contrário de todas as forças conhecidas da natureza, é puramente atrativa, assim ela năo pode ser enfraquecida por um plasma, como acontece no plasmá eletro magnético, por exemplo. É devido a esta propriedade que podemos detectar esta força extremarnente fraca, mas esta mesma propriedade a toma instävel.

Em 1902, Sir James Jeans mostrou, usando a teoria newtoniana clássica, que um fluido estatitico homogêneo näo-relativistico é instavel se perturbado por deformaçöes de comprimento de onda suficientemente longos. Considere um fuido com densidade $\rho$, velocidade $\vec{v}$, pressão $p$ e campo gavitacional $\vec{g}$ que satisfaz a equaçāo de continuidade:

$$
\frac{\partial p}{\partial t}+\vec{\nabla} \cdot(\rho \hat{)})=0
$$

a equação de Ềuer:

$$
\frac{\partial \vec{v}}{\partial t}+(\vec{v} \cdot \vec{\nabla}) \vec{v}=-\frac{1}{\rho} \vec{\nabla} p+\vec{z}
$$

e as equaçöes newtonianas para o campo gravitacional gerado pelo fluido:

$$
\begin{aligned}
\vec{\nabla} \times \vec{g} & =0 \\
\vec{\nabla} \cdot \vec{g} & =-4 \pi G p .
\end{aligned}
$$


onde $G$ a constante de gravitação universal.

Considendo agora pequenas perturbações $\delta \rho, \delta \vec{v}, \delta \vec{g}$ e $\delta p$ em torno da soluçăo estática, isto $\hat{e}$, da soluçẫo com $\rho$ e $p$ constantes e $\vec{v}$ e $\vec{g}$ nulos obtemos, em primeira ordem, as equaçoes (4.1)-(4.3) da forma:

$$
\begin{aligned}
\frac{\partial \delta \rho}{\partial t}+\rho \vec{\nabla} \cdot \delta \vec{v} & =0 \\
\frac{\partial \delta \vec{v}}{\partial t} & =-\frac{v_{s}^{2}}{\rho} \vec{\nabla} \delta \rho+\delta \vec{g} \\
\vec{\nabla} \times \delta \vec{g} & =0 \\
\vec{\nabla} \cdot \delta \vec{g} & =-4 \pi G \delta \rho
\end{aligned}
$$

onde $v_{*}^{2}$ é a velocidade do som:

$$
u_{*}^{z}=\frac{\partial p}{\delta p}
$$

Estas equaçöes podem ser combinadas para se obter uma equação diferencial para $\delta p$ da forma:

$$
\frac{\partial^{2} t \rho}{\partial^{2} t}-v^{2} \vec{\nabla} \delta p=4 n C p d p
$$

Note que o segundo membro da equação (4.6) tem a forma de um termo de massa, porém com o sinal trocado. Assim a soluçäo desta equação tem a forma:

$$
\delta p=C \exp (\vec{a} \cdot \vec{x}-i \omega t)
$$

onde

$$
\omega=\sqrt{k^{2} \eta_{s}^{2}-4 \pi G p}
$$

Desta forma, para quaisquer condiçôes iniciais e condiçôes contorno, com excessāo de um conjunto de medida nula, a soluçä̃o da equaçäo (4.6) possui uma parcela que cresce exponencialmente quando $k$ for menor que $k_{J}$ definido da forma:

$$
k_{I}=\sqrt{\frac{4 \pi G \rho}{v_{s}^{2}}}
$$

Esta instabilidade reffete que um fuido homogeneo espalhado por uma vasta regiāo do espaço é instavel se considerarmos o campo gravitacional. 
É importante notar que este resultado se refere a gravitaçäo newtoniana, $e$, neste contexto, podemos interpretar este resultado como sendo o motivo pelo qual se formam as galáxias, concentraçöes raaiores de matéria espacial, enquanto a regiäo entre elas é praticamente vazia. Esta instabilidade está intirnamente ligada so caráter puramente atrativo da força gravitacional, que faz corn que o plasma, em vez de atenuar, acentue a flutuaçäo da clensidade de massa, amplificando assim as perturbaşōes. Na formulaçào de Einstein da gravitaçäo, estas instabilidades são ainda majores, pois é possivel a formação de buracos negros que, uma vez formados, näo podern ser destruídos. Realmente, se uma estrela tiver massa maior que o limite de OppenheimerVolkoff, atualmente avaliado em 1.4 massas solares, esta estrela colapsará progressivamente até se tornar um buraco negro.

Dada a instabilidade da gravitação, pode-âe perguntar qual é o estado fundamental desta teoria, uma vez que a ação de Eintein nảo ê positiva definida mesmo quando euclidianizada, pois a curvatura pode ser arbitrariamente grande, positiva ou negativa. Desta forma a formulaçăo quântica da gravitaçāo nāo está bem definida, o que pode parecer que o estado fundamental da gravitaçāo quântica simplesmente não existe. Entretanto provon-se que, para espaços assintoticamente planos, a energia total definida em termos do comportanento assintótico dos campos gravitacionais é positiva semidefini$\mathrm{da}$, e o espaço de Minkowsky é o único espał̧o de energia zero. Isto implica que a temperatura zero o espaço de Minkowsky é estável e não pode decair por nenhum mecanismo. Para qualquer temperatura não nula, entretanto, o espaço de Minkowsky é instavel e vai decair.

Outro grande problema da gravitaçầo quântica é que, ao contrário das outras forças básicas da natureza, a gravitaçẳo nāo é renormalizável, pois sua constarte de acoplamento $G$ não é adímensional, como nas outras teorias. De fato, podemos obter uma unidade fundamental de comprimento, o comprimento de Planck. Desta forma, se estivermos estudando efeitos gravitacionais abaixo da escala de Planck, não podemos usar a teoria de perturbaçōes. A princípio parece que se estivermos acina da escala de Planck este problema estará resolvído, porém, de acordo corn o princípio equivalência, que é um dos princípios básicos desta teoria, todos os tipos de energia interagern de forma igualmente forte com a gravitaçāo, inclusive a própria energia gravitam cional. Ern linguagem da rnecânica quäntica, podemos dizer que um gráviton é tão sujeito ao campo gravitacional quanto, por exemplo, um fóton. Apesar destes problemas, podemos proceder de uma forma semi-clássica, impondo 
pequenas ascilagöes em tomo de urua métrica clássica[s]. 0 ponto de partida para o estudo da gravitação é a definição da métrica $g_{p}$ do espaço:

$$
d s^{2}=g_{1, y} d x^{3} d t^{2}
$$

onde $d s$ a distãncia entre dois pontos que possuem uma diferença $d \tau^{l t}$ em suas coordenadas. A derivada de um vetor:

$$
A_{i s, k} \equiv \frac{d A_{i t}}{d x^{t}}
$$

am geral näo se comporta como um tensor por uma mudança de coordenadas, porén a derivada covariante definida como:

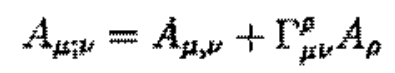

se comporta. Nesta formula $\mathrm{C}$ e simbolo de Chistoffel definido como:

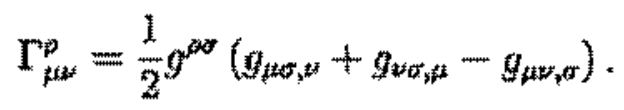

Desta forma podernos definir o tensor de curvatura $R^{\sigma}{ }_{p \sim \rho}$ como o comutador das derivadas covariantes, isto t:

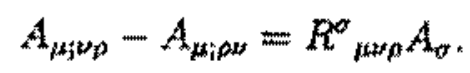

A partir deste tensor podemos definir o tensor de Ricci $R_{\mu \mu}=R_{\mu \rho \nu}$ e a murvatora escalar $R=g^{j v} R_{\mu \nu}$. Queremos agora obter uma lagrangeana de uma partícula esalar em urr espaço curvo, porém esta lagrangeana näo é unicamente determinada. De fato, qualquer lagrangeana definida como:

$$
\mathcal{L}(x)=-\frac{1}{2} \sqrt{-g(x)}\left(g^{\mu \nu} \partial_{\mu} \phi \partial_{\mu} \phi-\xi R \phi^{2}\right)
$$

onde $\xi$ é uma constante se reduz a langrangeana de uma partícula salar sob a métrica de Minkowsky. Porém um caso de interesse especial é quando a teoria é invariante conforme, isto $\varepsilon$, quando a teoria é invariante por ura mudança local de escala da forma:

$$
g_{\mu \nu} \rightarrow \Omega^{2}(x) g_{\mu \nu}
$$


Todas as forças básicas da natureza, com execẫo da gravitaçăo, sâo in variantes por esta transformaçāo, quando envolyem particulas nāo massivas, por isto vamos estudar o caso em que uma teoria escalar pode também ser invariante. Quando aplicanos uma transiomnaçäo a forma (4.16) pode se mastrar que o tensor de curvatura em 4 dimensogs se transforma como:

$$
R \rightarrow \Omega^{-2} R+6 \Omega^{-3} \mathrm{C} \Omega
$$

ande $\square \Omega$ \& definido da forma:

$$
w \Omega \equiv \frac{1}{\sqrt{-g}} \partial_{\mu}\left(\sqrt{-g} g^{\mu *} \partial_{\nu} n\right)
$$

Se impusermos também que o campo escalar $\phi$ se transíorma da forma:

$$
\rightarrow \Omega^{-1} \phi
$$

obteremos que a lagrangeana vai se transformar como:

$$
\left.\mathcal{L} \rightarrow \mathcal{L}+(6 \xi-1) \sqrt{-g} g^{\mu t z} \partial_{m} t \Omega \partial_{\nu}\left(\Omega^{-1} \phi^{2}\right)-6 \xi \partial_{\mu \hbar}(\sqrt{(}-g) \phi^{2} \Omega^{-1} g^{\mu \nu} \partial_{\nu} \Omega\right)
$$

de onde é fácil ver que se quisermos uma teoria invariante conforme temos de impor que $\xi=\frac{1}{6}$, pois desta forma a lagrangeana será acrecida apenas de uma derivada total, o que näo altera a aça. Como estamos estudando o caso en que as futuaçôes a métrica são pequenas variaçổes da métrica clássica de Minkowsky, a métrica de nosso espaço sera:

$$
g_{\mu t}=\eta_{\mu \nu}+\kappa h_{\mu \nu}
$$

onde $\eta$ é a métrica de Minkowsky, $h$, as flutuçöes e $k$, a densidade de energia do sistema.

Assim como a invariância de gauge do eletromagnetismo impōe as identidades de Ward, a invariancia conforme impöem relaços entre as funçōes de Green do gráviton. Para entender isto varnos fazer uma transformação infinitesimal do tipo:

$$
\Omega=1+\delta \omega_{*}
$$

Com esta transformação o campo $h$ definido em (4.21) se transformara da forma:

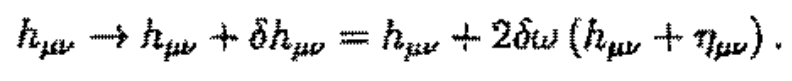


Assim a açầ com as fontes do campo $h$ definida como:

$$
S_{J}=\int d^{4} x\left(\mathcal{L}+J^{j w} h_{p u r}\right),
$$

se transformará da forrna:

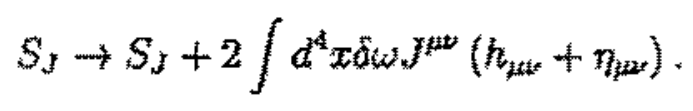

Desta forma ê fâcil ver que o gerador das funôes de Green definido como:

$$
e^{G(J)}=\int \mathcal{D} h \mathcal{D} \phi e^{i S_{J}},
$$

deverá obedecer à identidade:

$$
\left[\eta_{\nu \nu}+\frac{\delta}{\delta J u}\right] e^{\sigma(n)}
$$

Esta identidade é conhecida como identidade de de Weyl.

Quando a temperatura está suficientemente abaixo da escala de Planck, a teoria de perturbaçấo pode ser aplicada para calcular os efeitos tếrnicos desta teoria. Com isto podemos obter as regras de Feynman expandindo a açäo em série de $h$, lembrando-se que os vértices desta teoria envolvem duas partículas escalàres e um número arbitrário de grávitons. $O$ vértice com um gráviton é dado pela fórmula:

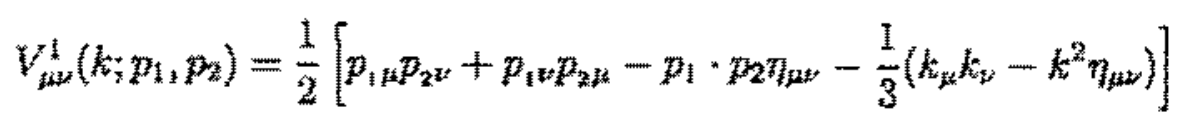

onde $k$ é o momento do gráviton e $p$ s os momentos das partículas escalares. $O$ vértice corn dois grávitons é consideravelmente mais conplexo:

$$
\begin{aligned}
& V_{\mu \nu, \alpha \beta}^{2}\left(k_{1}, k_{2} ; p_{1}, p_{2}\right)=\frac{1}{16}\left[-8 p_{1} p_{2 \beta} \beta \eta_{\alpha \mu}+2 p_{1} \cdot p_{2} \eta_{\alpha \mu} \eta_{\beta \mu \nu}+\right. \\
& { }_{4} p_{2 \alpha p_{2} \beta} \eta_{\mu \nu}-p_{1} \cdot p_{2} \eta_{\alpha \beta} \eta_{\mu \nu}+ \\
& \frac{1}{3}\left(2 k_{1 \mu} k_{1 \mu} \eta_{\alpha \beta}+2 k_{1 \mu} k_{3 \nu} \eta_{\alpha \beta}-2 k_{1} \beta k_{1}, \eta_{\alpha \beta}-\right.
\end{aligned}
$$

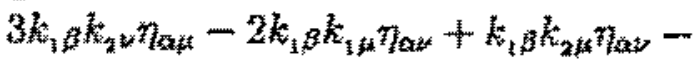

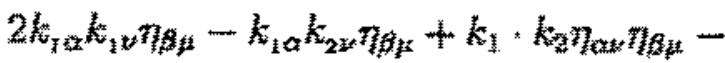

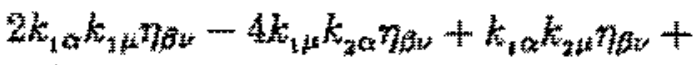

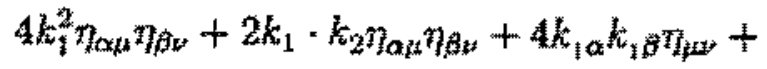

$$
\begin{aligned}
& \left.\left.2 k_{1 \beta} \beta k_{2 \alpha} \eta_{\mu \nu}-2 k_{1}^{2} \eta_{\alpha \beta} \eta_{k \alpha+}-k_{1} \cdot k_{2} \eta_{\alpha \beta} \eta_{\alpha \omega \nu}\right)\right]
\end{aligned}
$$


Vêrtices de mais grávitons são extreranarnente complicados, porém podern ser obtidos par um cảlculo sistemático.

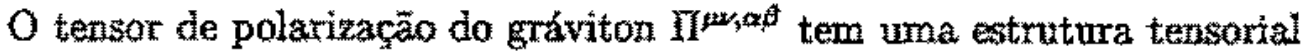
muito mais complexa do que no caso da $\mathrm{QCD}$, pois ele tem de ser simétrico erra relaçầ à troca de índices $\mu \leftrightarrow \nu$ ou $\alpha \rightarrow \beta$ e a troca de pares de índices $(\mu \nu) \leftrightarrow(\alpha \beta)$. Com isto podemos escrever o tensor de polarizaçio em termos de 14 tensores independente da forma:

$$
\mathrm{M}^{\mu \nu, \alpha \beta}=\sum_{i=1}^{14} \Pi_{i} T_{i}^{\mu \nu, \alpha \beta}\left(\nu_{i}, \ldots\right)
$$

onde $T_{\{} \ldots, \alpha \beta(X, Y)$ sảo dados pela tabela:

$$
\begin{aligned}
& T_{1}^{\mu \nu \alpha \beta}(X, Y)=\eta^{\alpha \nu} \eta^{\beta \mu}+\eta^{\alpha \mu \eta_{1} \eta^{\beta \nu}} \\
& T_{2}^{\mu \nu, \alpha \hat{p}}(X, Y)=X^{\mu}\left(X^{\hat{\beta}} \eta^{\alpha \nu}+X^{\alpha \alpha} \eta^{\beta \nu}\right)+X^{\nu}\left(X^{\beta} \eta^{\alpha z}+X^{\alpha \alpha} \eta^{\beta \mu}\right) \\
& T_{3}^{2 z_{i} \alpha \beta}(X, Y)=X^{\alpha} X^{\beta} X^{\mu} X^{\nu} \\
& T_{i}^{\alpha+\alpha, \alpha \beta}(X, Y)=\eta^{\alpha \beta} \eta^{\mu \nu}
\end{aligned}
$$

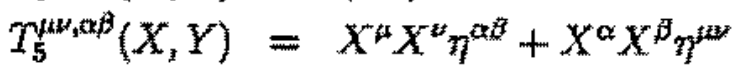

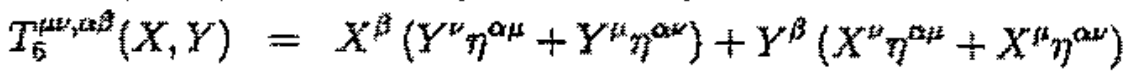

$$
\begin{aligned}
& +X^{\alpha}\left(Y^{\nu} \eta^{\beta \mu}+Y^{\mu} \eta^{\beta \nu}\right)+Y^{\alpha}\left(X^{\nu} \eta^{\beta \mu}+X^{\mu} \eta^{\beta \nu}\right) \\
& T_{7}^{\alpha \nu, \alpha a}(X, Y)=Y^{\nu} X^{\alpha} X^{\mu} X^{\mu}+Y^{\mu} X^{\alpha} X^{a} X^{\nu}+Y^{\beta} X^{\alpha} X^{z} X^{\nu} \\
& +Y^{\alpha} X^{b} X^{\mu} X^{\mu}
\end{aligned}
$$

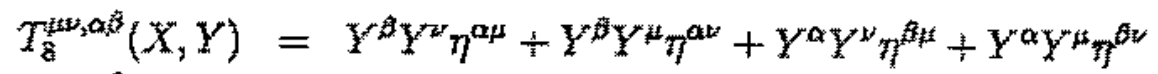

$$
\begin{aligned}
& T_{\beta}^{\mu \nu, \alpha \beta}(X, Y)=Y^{\mu} Y^{\nu} X^{\alpha} X^{\beta}+Y^{\alpha} Y^{\beta} X^{\beta} X^{z} \\
& T_{10}^{\mu \alpha, \alpha}\left(X_{1} Y\right)=\left(Y^{\beta} X^{\alpha}+Y^{\alpha} X^{\beta}\right)\left(Y^{z} X^{\mu}+Y^{\mu} X^{\mu}\right)
\end{aligned}
$$

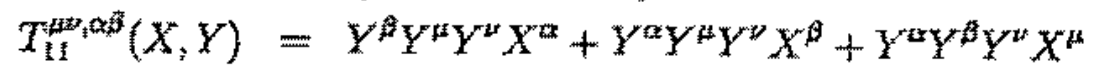

$$
\begin{aligned}
& +Y^{\alpha \alpha} Y^{\beta} Y^{\beta} X^{*} \\
& T_{12}^{\mu \nu, \alpha \beta}(X, Y)=Y^{\alpha} Y^{\beta} Y^{\mu \alpha} Y^{\nu}
\end{aligned}
$$

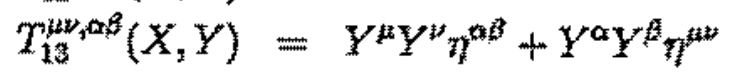

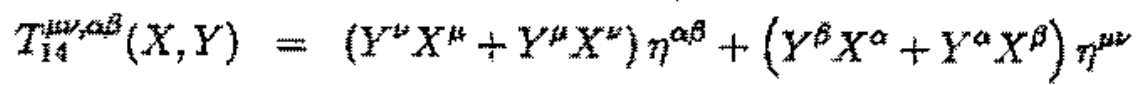

A identidade de Weyl (4.27) impōe para o tensor de polariaçâa a identi- 
dade:

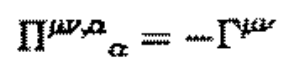

onde $\Gamma^{\mu s}$ ê a funçäo de um ponto do gráciton, o tadpole". Por construçăo, eta teoria ể invariante, também, por uma tranformaç̄o geral de coordenadas, fazendo com que o tensor de polarização obedeça tambèm a identiỏade de Wart:

$$
2 k_{\nu} \Pi^{\mu \mu_{1} \alpha \beta}(k)=k^{\mu} \Gamma^{\alpha \beta}-k_{c}\left(\Gamma^{\alpha \sigma^{\alpha}} \eta^{\beta \mu}+\Gamma^{\beta \alpha} \eta^{\alpha \mu}\right)
$$

A temperatura finita o tensor de polarizaçấo nāo é invariante por uma reparametrizaçäo do campo gravitacional, isto ê, se no lugar da parametrização (4.21) usassemos outra, por exemplo:

$$
\sqrt{-g g^{z \nu}}=\eta^{\mu \nu}+\phi^{\mu z}
$$

o tensor de polarizaçăo seria diferente. Isto se deve ao fato do tadpole nato se anular. Pelas identidades de Ward a funça de 1 ponto deve ter traço nulo, que a temperatura zero implica em que seja nulo, porém a temperatura finita implica somente que de deve ser proporcional a $4 \eta^{20} \eta^{20}-\eta^{\mu 2 \nu}$. Para entender melhor este ponto, considere a aça efetiva, que gera as funçōes de Green, em uma parametrizaçăo qualquer:

$$
S_{\mathrm{eff}}=\Gamma^{\alpha \beta} \phi_{\alpha \beta}+\frac{1}{2} \int d^{\mathrm{d}} \eta^{m, \alpha \beta}(k) \phi_{\mu \alpha}(k) \phi_{\alpha \beta}(k)+O\left(\phi^{3}\right)
$$

Se fzermos uma reparametrizaça, o que corresponde a:

$$
\phi_{\mu \nu} \rightarrow \pm \phi_{\mu \mu}+\eta_{\mu \nu} \phi_{\lambda}^{\lambda}+O\left(\phi^{2}\right)
$$

obteremos novos termos quadráticos em $\phi$, o que corresponde a uma mudança no valor de II. Porém facil ver, usando a tdentidade de Weyl (4.32), que todos estes novos termos são proporcionatis a $\Gamma$. Assim, para obtermos a parte invariante de $\mathrm{II}$ devemos retirar a dependeneia deste com o tadpole. Isto é possivel definindo-se:

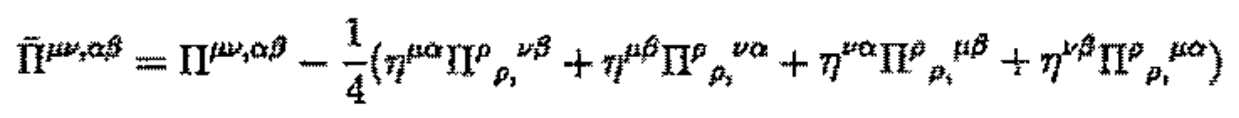

Notando que os termos provenientes do tadpole por uma reparametrizacäo säo independentes do momento e que devem obedecer a identidade de Ward (4.33), ếâcil ver que a açäo efetiva näo gerará novos termos quadráticos em 申que contribuam para $\overrightarrow{\hat{\mathbf{n}}}$. 


\section{Capítulo 5}

\section{Efeitos de campos térmicos no tensor de polarização do gráviton}

Varnos calcular o tensor de polarizaçào do gráviton utilizando o método de espalhamento frontal exposto no primeiro capítulo. Independente do campo que pusermos para interagir com o gräviton, em primeira ordem de perturbaç̃̃o este tensor terá a estrutura geral da forma:

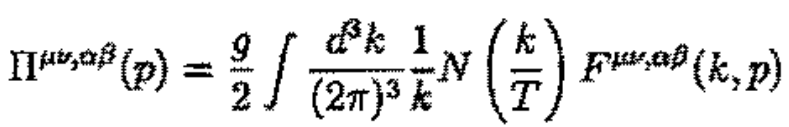

onde $F$ a amplitude de espalhamento frontal e $y$ é uma constante que conta os graus de liberdade do campo $(g=1$ para partículas escalares, $y=2$ para a radiaçâo, etc.) As simetrias por troca de indices de $F$ são as mesmas que as de $\Pi$, porem, por se tratar de uma amplitude de espalhanento ele tem de ser invariante de Lorentz. Assim ele pode ser representado por tensores como os da tabela $(4,31)$ com $(X, Y) \rightarrow(k, p)$. Usando-se esset tensores podemos escrever:

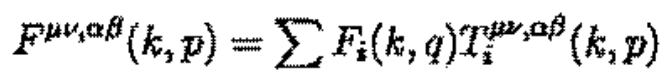

Vamas primeirraente analisar vínculos que aparecem en $F_{i}$ se impuermos as identidades de Ward (4.33) e Weyl (4.32). Usando que a estrutura geral 
do tadpole é representada por:

$$
\Gamma^{\alpha \beta}=\frac{g}{2} \frac{\kappa}{(2 \pi)^{3}} \int \frac{d^{3} k}{k} N\left(\frac{k}{T}\right) k^{\alpha} k^{\beta}
$$

Desta forma as identidades de Ward e Weyl para $F$ impõem:

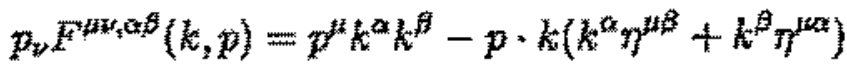

$$
\begin{aligned}
& F_{\alpha}^{* v_{1} \alpha \alpha}(k, p)=-2 k^{\alpha \mu} k^{* t}
\end{aligned}
$$

Estas identidades representam um grande vinculo para $F$; equaçäo (5.4) resulta em 10 vínculos e a equação $(5.5)$ em outros 4 . Porén estas

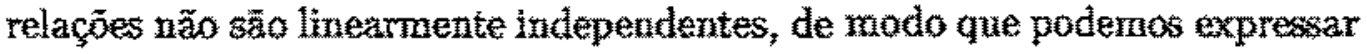
11 funções $H_{\text {i }}$ em funça das outras trè desta forma:

$$
\begin{aligned}
& F_{1}=\frac{3 p^{4}}{4} F_{12}+p \cdot k F_{14} \\
& F_{2}=-\frac{1}{2}-\frac{p^{4}}{2 p \cdot k} F_{11}+\frac{p^{2}}{p \cdot k} F_{14} \\
& F_{3}=-\frac{p^{2}}{2(p \cdot k)^{2}}-\frac{2 p^{6}}{(p \cdot k)^{3}} F_{11}+\frac{p^{4}}{(p \cdot k)^{3}} F_{14} \\
& F_{4}=-\frac{p^{4}}{2} F_{12}-(p \cdot k) F_{14} \\
& F_{1}=-\frac{p^{2}}{(p \cdot k)} F_{14} \\
& F_{6}=\frac{p^{2}}{2} F_{11}-F_{14} \\
& F_{7}=\frac{1}{2 p \cdot k}+\frac{2 p^{4}}{(p \cdot k)^{2}} F_{11}-\frac{p^{2}}{(p \cdot k)^{2}} F_{14} \\
& F_{8}=-\frac{p \cdot k}{2} F_{11}-\frac{3 p^{2}}{4} F_{12} \\
& F_{3}=-\frac{2 p^{2}}{p \cdot k} F_{11}+\frac{3}{p \cdot k} F_{14} \\
& F_{10}=-\frac{3 p^{2}}{2 p \cdot k} F_{11} \\
& F_{13}=\frac{p^{2}}{2} F_{12}
\end{aligned}
$$


Note que as identidades de Ward e Weyl näo fixam as funçöes $F_{t 1}, F_{12}$ e $F_{14}$ : porérn as propriedades da amplitude de espalbamento frontal podern nos fornecer mais vínculos. Como vimos no primeiro capítulo, esta amplitude é simêtrica em $p \leftrightarrow-p$ e, na ordem de um loop, tern no máximo um denominador da forma $p^{3} \pm 2 k \cdot p$. Além disto, por análise dimensional ${ }^{3}$ facil ver que $F^{\mu \omega, a \beta}$ tem dimensão de (momento) ${ }^{2}$. Estas condições implicam que o mumerador das funçöes $F_{3}$ e $F_{7}$ seja apenas uma constante adimensio nal, pois estas componentes săo multiplicadas pelos tensores $T_{3}$ e $T_{7}$ que têm dimensäo de (momento) ${ }^{2}$. Assim podernos escrever:

$$
\begin{aligned}
& F_{3}=c_{3}\left(\frac{1}{p^{2}+2 p \cdot k}+\frac{1}{p^{2}-2 p \cdot k}\right) \\
& F_{7}=c_{7}\left(\frac{1}{p^{2}+2 p \cdot k}-\frac{1}{p^{2}-2 p \cdot k}\right)
\end{aligned}
$$

onde $c_{i}$ são constantes. Além disto, notando que:

$$
\frac{p \cdot k}{p^{2} \pm 2 p \cdot k}= \pm \frac{1}{2}\left(1-\frac{p^{2}}{p^{2} \pm 2 p \cdot k}\right)
$$

podemos escrever $F_{2}$ da forma:

$$
F_{2}=c_{2} k \cdot p\left(\frac{1}{p^{2}+2 p \cdot k}-\frac{1}{p^{2}-2 p \cdot k}\right)+c_{2}^{\prime} p^{2}\left(\frac{1}{p^{2}+2 p \cdot k}+\frac{1}{p^{2}-2 p \cdot k}\right)
$$

Aplicando isto exn (5.6) podemos fixar unicamente as constantes $c_{2}, c_{3} \mathrm{e}$ cr obtendo:

$$
c_{2}=-\frac{1}{2} ; \quad c_{3}=1 ; \quad c_{7}=\frac{1}{2}
$$

Estas relaçöes permitem escrevermos:

$$
F_{14}=2 p^{2} F_{11}+\frac{p^{2}(p \cdot k)}{2\left[p^{4}-4(p . k)^{2}\right]}
$$

desta forma sobrarn apenas duas funçöes independentes, as funçöes $F_{11} \in F_{12}$, que tem de ter as estruturas:

$$
F_{11}=c_{11}\left(\frac{1}{p^{2}+2 p \cdot k}-\frac{1}{p^{2}-2 p \cdot k}\right)
$$




$$
F_{12}=c_{12}\left(\frac{1}{p^{2}+2 p \cdot k}+\frac{1}{p^{2}-2 p \cdot k}\right)
$$

onde $c_{11}$ e $c_{12}$ que permenecem indeterminadas. Vale ressaltar que stats propriedades valem para qualquer teoria que seja invariante conforme, pois usamos somente as identidades de Ward e Weyl e astrutura do tadpole.

Os termos dominantes exn altas temperaturas seräo os termos dominantes na amplitude de espalhamento frontal quando $k \rightarrow \infty$. Com isto, é facil ver que os únicos termos que irāo contribuir sāo os termos $F_{2}, F_{3}$ \& $F_{7}$. Como vimos a parte dominante destes termos é unicamente determinada, ou sejo, é independente da natureza das particulas térnicas a menos de uma constante multiplicativa. Estes termos podem ser integrados expandindo-se os denominadores de $F$ da forma:

$$
\frac{1}{p^{2}+2 p \cdot k}=\frac{1}{2 p \cdot k}-\frac{p^{2}}{(2 p \cdot k)^{2}}+\mathcal{O}\left(k^{-1}\right)
$$

usando-se as integrais:

$$
\int_{0}^{\infty} k^{z} d k N\left(\frac{k}{T}\right)=T^{x+1} T(x+1) \zeta(x+1)
$$

obtendo-se assim:

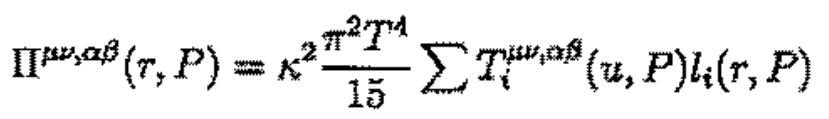

onde foram utilizadas as definições:

$$
r \equiv \frac{p_{0}}{|\vec{p}|} ; \quad P^{\prime s} \equiv(r, \hat{p})
$$

e as funçö $l_{i}$ sầ dadas por:

$$
\begin{aligned}
& l_{1}=\frac{1}{6}-\frac{P^{2}}{24}+\frac{P^{4} L}{8} \\
& l_{2}=-\frac{1}{3}+\frac{P^{2}}{12}-\frac{5 P^{4}}{24}+\frac{5 P^{6} L}{8} \\
& l_{3}=\frac{-P^{2}}{3}+\frac{7 P^{4}}{12}-\frac{35 P^{4}}{24}+\frac{35 P^{8} L}{8} \\
& l_{4}=\frac{-P^{2}}{24}+\frac{P^{4} L}{8}
\end{aligned}
$$




$$
\begin{aligned}
& l_{5}=\frac{P^{2}}{12}-\frac{5 P^{4}}{24}+\frac{5 P^{6} L}{8} \\
& l_{5}=\left(\frac{-1}{12}+\frac{5 P^{2}}{24}-\frac{5 P^{4} L}{8}\right) r \\
& l_{7}=\left(\frac{1}{3}-\frac{7 P^{2}}{12}+\frac{35 P^{4}}{24}-\frac{35 P^{6} L}{8}\right) r \\
& l_{8}=-\frac{1}{12}-\frac{5 P^{2}}{24}+\frac{P^{2} L}{2}+\frac{5 P^{4} L}{8} \\
& l_{9}=\frac{1}{6}-\frac{2 P^{2}}{3}-\frac{35 P^{4}}{24}+\frac{15 P^{4} L}{4}+\frac{35 P^{6} L}{8} \\
& l_{10}=\frac{1}{6}-\frac{2 P^{2}}{3}-\frac{35 P^{4}}{24}+\frac{15 P^{4} L}{4}+\frac{35 P^{6} L}{8} \\
& l_{11}=\left(\frac{1}{4}+\frac{35 P^{2}}{24}-\frac{5 P^{2} L}{2}-\frac{35 P^{4} L}{8}\right) r \\
& l_{12}=-\frac{13}{12}-\frac{35 P^{2}}{24}+L+5 P^{2} L+\frac{35 P^{4} L}{8} \\
& l_{13}=-\frac{1}{12}-\frac{5 P^{2}}{24}+\frac{P^{2} L}{2}+\frac{5 P^{4} L}{8} \\
& l_{14}=\left(\frac{-1}{12}+\frac{5 P^{2}}{24}-\frac{5 P^{4} L}{8}\right) r
\end{aligned}
$$

Vamos estudar agora o caso específico de partículas escalares. Neste caso temos:

$$
c_{11}=\frac{1}{12} ; \quad c_{12}=\frac{1}{36}
$$

Varnos calcular o tensor de polarizaçäo do gráviton de forma exata para partículas térmicas escalares. O terroo dominante em altas tempereturas é a fórmula (5.17), portanto estamos apenas interessados nos termos subdominantes dados pela fórmula:

$$
\Pi_{S_{i}}=\kappa^{2} \mid p^{3} N_{i}\left(r_{2} P\right)
$$

Para ísto fizemos a contraçäo:

$$
\vec{P}_{i}(r, P)=\frac{1}{8} \Pi^{\mu \mu, \alpha \beta}(k) T_{i, \mu, \alpha \beta}(u, P)
$$


Após isto, as funçöes $\Pi_{i}$ podern ser determinadas por:

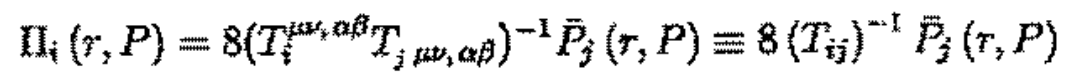

onde $\left(T_{i j}\right)^{-1}$ representa a matrix inversa a $T_{i j} \equiv T_{i} w_{1} \alpha \beta T_{j}, \mu \nu, \alpha \beta^{*}$

Pode-se observar, usando as identidades de Ward e Weyl, que a contraçäo de $\Pi$ com os tensores $T_{i}^{4 t, \alpha \beta}$ corn $i$ de 4 a 14 dão termos proporcionais a tadpole, logo independentes do momento e dominantes a altas temperaturas. Assim os únicos terroos sub-dominantes säo $\bar{P}_{1}, \bar{P}_{2}$ e $\bar{P}_{3}$. Quando calculamos o tensor de polarizaçäo observarnos que estes termos envolvem integrais da forma:

$$
I_{S}(p, T)=\frac{1}{(2 \pi)^{3}} \int \frac{d^{3} k}{2|k| \exp (|\vec{k}| / T)-1}\left(\frac{1}{p^{2}+2 p \cdot k}+\frac{1}{p^{2}-2 p \cdot k}\right)
$$

com $S=0,2$ ou 4 En termos de $=\cos (\theta)$, onde $\theta$ é o angulo entre $\vec{p}$ e $\vec{k}$, obtemas:

$$
I_{S}(p, T)=-\frac{1}{(2 \pi)^{2}} \frac{p^{2}}{4} \int_{-1}^{1} \frac{d x}{(p 0-|p| x)^{2}} \int_{0}^{\infty} d Q \frac{Q^{B+1}}{\exp (Q / T)-1} \frac{1}{Q^{2}+(2 \pi T y)^{2}}
$$

onde,

$$
y=\frac{1}{4 i n T} \frac{p^{3}}{p_{0}-|p| x}
$$

Além de funçōes elementares, as integrais da equaçã̃o (3.25) pode ser reduzida à integral básica da forma[11]:

$$
\begin{aligned}
I(y) & =\int_{0}^{\infty} \frac{Q d Q}{Q^{2}+(2 \pi T y)^{2}} \frac{1}{\exp (Q T T-1} \\
& =\frac{1}{2} \Theta[\operatorname{Re}(y)]\left(\log y-\frac{1}{2 y}-\psi(y)\right)+(y \leftarrow-y)
\end{aligned}
$$

de forma análoga to câlculo da teoria escalar com acoplamento $\lambda \phi^{3}$. Novamente nesta equação $\psi(y)=\frac{a}{d y} \ln \Gamma(y)$ e a função psi de Enler. $O$ limite de tempo real da funçäo de Green pode ser obtido com a continuçấo analítica do formalismo de tempo imaginário pela prescrição [16] $k_{0}=(1+i \varepsilon) K_{0}$ onde 
$\varepsilon \rightarrow 0^{+}$e $K_{0}$ é real. Com a presença do fator $i E$ na equaçă (5.27), nós temos que $\operatorname{Re}(y)=\varepsilon^{t} \operatorname{Re}\left(k_{0}\right)$, com $z^{t} \rightarrow 0^{+}$.

Muitas das integrais angulares em (5.25) padem facilmente serem resolvidas em termos de funçôes elementares após uma troca de variáveis de $x$ para $y$. As integrais mais complicadas envolvem ternos contendo $\psi(y)$ multiplicados por potências de $\}$. Estas integrais têrn a forma:

$$
J_{n} \equiv \Theta\left[\operatorname{Re}\left(k_{\theta}\right)\right]\left[J_{n}\left(t\left(k_{0}\right)\right)-J_{n}\left(-t\left(-k_{0}\right)\right]\right]+\left[k_{0} \leftrightarrow-k_{0}\right]
$$

onde

$$
y_{n}(t)=\left(\frac{\operatorname{tm} T}{|\vec{h}|}\right)^{n+1} \int_{C}^{t} y^{n}\left[\ln (y)-\frac{1}{2 y}-\psi(y)\right] d \psi_{1}
$$

de forrna totalnente análoga ao resultado do primeiro capitulo. A scolha de $C$ é irrelevante, pois qualquer constante de integração que porventura apareça cancelará na expressão (5.28). Os valores $\left(h_{0}\right)$ e $-t\left(-h_{0}\right)$ são os limites de integracăo da integral em $y$ referentes aos valores $x=1$ e $x=-1$, respectivamentes, na equacão $(5,26)$. Logo:

$$
t\left(k_{0}\right)=\frac{k_{0}+|\vec{k}|}{\alpha_{k}+\pi T}
$$

Estas integrats podem ser expressas de forma fechada am termos de derivadas da função zeta de Riemann generalizada $\zeta(-n, t)$ onde $n$ é um numero inteiro de 0 a 4. Por exemplo, podemos obter:

$$
\begin{aligned}
J_{0}(t) & =\frac{|\vec{k}|}{i \pi T} \int^{t}\left[\ln (y)-\frac{1}{2 y}-\psi(y)\right] d y \\
& =\frac{|\vec{k}|}{i \pi T}\left[t \ln t-\zeta^{2}(0, t)-t-\frac{1}{2} \ln t\right] \\
J_{1}(t) & =\int^{t} y\left[\ln (y)-\frac{1}{2 y}-\psi t(y)\right] d y \\
& =t C^{t}(0, t)-C^{2}(-1, t)+\frac{t^{2}}{2} \ln t-\frac{3}{4} t^{2}
\end{aligned}
$$

Nas equaçöes acima, a derivada da funçäo zeta telativa ao primeiro atgumento. As funço $J_{n}(t)$ săo apresentadas no apendice A. Desta forma, 
vemos que as funçoes $P_{j}(j=1,2,3)$ na equaçāo (5.22) estäo relacionadas com $J_{n}$ definido como $(5,28)$ da seguinte forma

$$
\begin{aligned}
\tilde{P}_{1}(r, K) & =-\frac{K^{2}}{96}-\frac{K^{4}}{32} J_{0} \\
\bar{P}_{2} & =\frac{K^{2}}{48} L(r)-\frac{K^{4}}{32} J_{0}-r \frac{K^{2}}{4} J_{1}-\frac{K^{2}}{2} J_{2,} \\
\vec{P}_{3} & =-\frac{1}{576}+\frac{K^{2}}{192} L(r)-\frac{K^{4}}{256} J_{0}-r \frac{K^{2}}{16} J_{1}-\frac{2+3 K^{2}}{8} J_{2}-r J_{3}-J_{45}
\end{aligned}
$$

onde usou-se a definiçä:

$$
L(r)=\frac{r}{2} \ln \frac{r+1}{r-1}-1
$$

Assim, as funcöes $N_{i}(r, K)$ podern ser explicitamente determindas pelas equaçốes (15.21) e (5.23). Fazendo estas contas, obtemos:

$$
\begin{aligned}
& N_{1}(r, K)=K_{1}+K^{2} P_{2}+K^{4} P_{3} \\
& N_{2}(r, K)=K^{2} P_{1}+2 K^{4} P_{2}+5 K^{6} P_{3} \\
& N_{3}(r, K)=K^{4} P_{1}+5 K^{6} P_{3}+35 K^{8} P_{3} \\
& N_{4}(r, K)=-P_{1}-K^{2} P_{2}+K^{4} P_{3} \\
& N_{5}(r, K)=-K^{2} P_{1}-K^{4} P_{2}+5 K^{5} P_{3} \\
& N_{6}(r, K)=-r\left(P_{1}+2 K^{2} P_{2}+5 K^{4} P_{3}\right) \\
& N_{7}(r, K)=-r\left(K^{2} P_{1}+5 K^{4} P_{2}+35 K^{6} P_{3}\right) \\
& N_{8}(r, K)=P_{1}+\left(1+2 K^{2}\right) P_{2}+\left(4 K^{2}+5 K^{4}\right) P_{3} \\
& N_{9}(r, K)=\left(2+K^{2}\right) P_{1}+\left(6 K^{2}+5 K^{4}\right) P_{2}+\left(30 K^{4}+35 K^{6}\right) P_{3} \\
& N_{10}(r, K)=K^{2} P_{1}+\left(3 K^{2}+5 K^{4}\right) P_{2}+\left(30 K^{4}+35 K^{6}\right) P_{3} \\
& N_{1}(r, K)=-r\left[P_{1}+\left(2+5 K^{2}\right) P_{2}+\left(20 K^{2}+35 K^{4}\right) P_{3}\right] \\
& N_{12}(r, K)=P_{1}+\left(4+5 K^{2}\right) P_{2}+\left(8+40 K^{2}+35 K^{4}\right) P_{3} \\
& N_{13}(r, K)=-P_{1}-K^{2} P_{2}+\left(4 K^{2}+5 K^{4}\right) P_{3} \\
& N_{4}(r, K)=r\left(P_{1}+K^{2} P_{2}+5 K^{4} P_{3}\right)
\end{aligned}
$$


En altas temperaturas, funçăo zeta generalizada pode ser expandida em série de potencias de $t$, como é mostrado na equaçăo (A.11). Desta forma as funçôs $P_{j}(j=1,2,3)$ podern ser expressas em séries de potências de $(1 / T)$. Os termos dominantes destas séries säo dados por:

$$
\begin{aligned}
& P_{1}=-\frac{K^{2}}{96} \\
& P_{2}=\frac{K^{2}}{48} L(r) \\
& P_{3}=-\frac{1-3 K^{2} L(r)}{576}
\end{aligned}
$$

Apesar destas contribuiçōes serern invariantes de gauge, elas năo escrevem propriedades fisicas do plasma a tempertara finita. Em teoria de carnopos a ternperatura finita, as contribuçôes irreduciveis por uma partícula dependem, geralmente, da parametrização do campo gravitacioal. Isto se deve ao fáto da contribuiçẫo do "tadpole" nẫo se anular. Entretanto o tensor de traço nulo definido como:

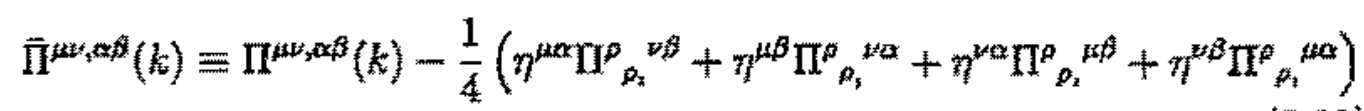

representa uma quantidade independente da parametrizaçăo do grăviton, como vinos no capitulo anterior. Desta forma, podemas estudar as massas têrroicas que aparecem no propagor do gráviton, de forma análoga ao estudo do segundo capítulo. onde:

O propagador clássico do gráviton pode ser escrito da forma $k^{-2} P_{\alpha \beta}^{\mu \prime \prime}$,

$$
P_{\alpha \beta}^{\mu \mu \nu}=\frac{1}{2}\left(\delta_{\alpha}^{\mu} \delta_{\beta}^{\nu}+\gamma_{\alpha}^{\alpha} \delta_{\beta}^{\mu} \eta^{\mu \nu} \eta_{\alpha \beta}\right) \equiv \mathbb{1}_{\alpha \alpha \beta}^{\mu \alpha \nu}-\frac{\eta^{\mu \nu} \eta_{\alpha \hat{\beta}}}{2}
$$

que é invariante por mudança na parmetrizaçäo dos campos.

$O$ propagador efetivo do gráviton pode ser obtido por sucessivas inserçōes de $\overline{\mathbf{I}}$ no propagador clóssico, obteado-se:

$$
\mathcal{D}_{\alpha \beta}^{i \nu}(k)=\frac{1}{k^{2}}\left[p_{\alpha \beta}^{\mu \nu}+n_{\alpha \beta}^{\mu \nu}+\left(\frac{1}{k^{2}}\right)^{2} \Pi_{p \alpha}^{\mu \nu} \Pi_{\alpha \beta}^{\rho \sigma}+\cdots\right] .
$$

Como este propagador envolve apenas quantidades invariantes por mudança na parametrização dos campos, ele possư significado físico, assim como as grandezas que forem obtidas através dele. A equaçato (5.41) pode ser 
somada como uma série geométrica, obtendo-se:

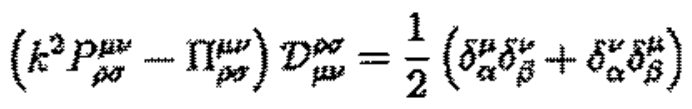

O propagador efetivo satisfaz alguns vinculas funamentais. Por exemplo, devido ace traco nulo de $\bar{\Pi}$, a equaçăo (5.41) impoônera que:

$$
\mathcal{D}_{p}^{\mu, \mu \nu}=-\frac{\eta_{\mu \nu}}{k^{2}}
$$

Alén disso, a identidade de Ward (4.33) impöe que:

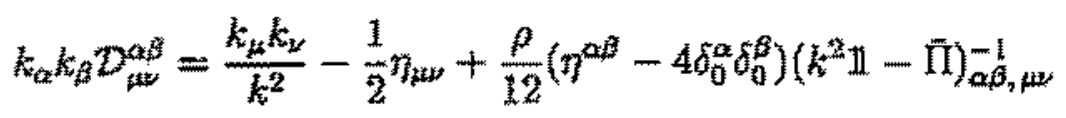

onde a densidade de energia $\rho$ é dada por:

$$
p=\frac{g \pi^{2} T^{4}}{30}
$$

Novarnente aqui 9 representa o número total de grans de liberdade das partículas têrmicas.

Como vimos no capítulo 2 , as mascias térmicas surgern quando $k_{0}=0$. Estas massas serão importantes para vermos a absorção do campo pelo plasma térmico. Quando elas são nulas, provocam divergências infravermelhas na teoria, como a parte magnética de uma teoria de Vang-Mills, engquanto se forem negativas denotam instabilidades, como a massa de Jeans clássica. Em $h_{0}=0$, a parte dominante em altas temperaturas de $\bar{\Pi}$ pode ser escrita da forma:

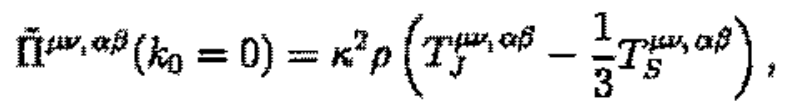

onde $T_{J}$ e $T_{S}$ sầo tensores definidos coma:

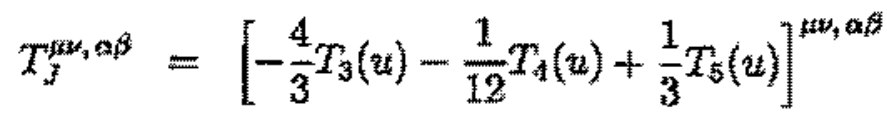

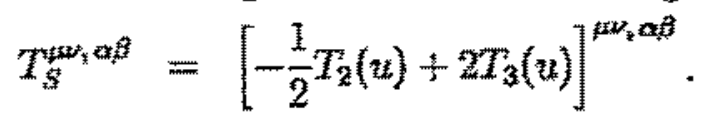

Note que estes tensores säo de traço nulo, idernpotentes (a menos de um sinal) e ortogonais entre si. Estes tensores säo os modos normais de vibraçäo 
do plasma. O modo descrito por $T_{J}$ é espacialmente longitudinal, enquanto o modo descrito por $T_{S}$ é espacialmente transversal, isto é:

$$
k_{i} k_{j} T_{S}^{i j_{3}, y}=0 ; \quad(i, j=1,2,3) .
$$

Desta forma, é fácil inverter a equaçāo (3.46), obtendo para o propagador efetivo do gráviton o resultado:

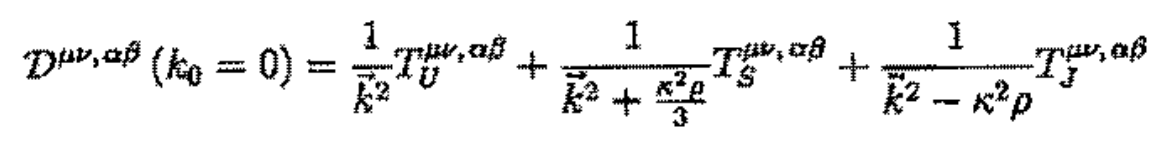

onde o terceiro modo normal $T_{U}$ é dado por:

$$
T_{U}^{\mu \nu, \alpha \beta}=\left[-\frac{1}{2} T_{1}(u)+\frac{1}{2} T_{2}(u)-\frac{2}{3} T_{3}(u)+\frac{7}{12} T_{4}(u)-\frac{1}{3} T_{5}(u)\right]^{\mu \nu, \alpha \beta},
$$

que é normal aos modos $T_{J}$ e $T_{S}$.

Podemos ver que a massa associada ao modo $T_{V}$ é nula, isto $e$, o plasma näo absorve nem amplifica ondas associadas a este modo, de modo análogo à parte magnética da QCD. Por outro lado, a massa associada ao modo $T_{S}$ é:

$$
m_{S}^{2}=\frac{\kappa^{2} \rho}{3}=\frac{32 \pi G \rho}{3}
$$

equivalente ao modo elétrico de um plasma de QCD, ou ao plasma da teoria escalar com acoplamento $\lambda \phi^{a}$.

$O$ modo $T_{J}$ é caracterizado por uma massa imaginária:

$$
m_{J}^{2}=-32 \pi G \rho
$$

de forma equivalente à massa de Jeans clássica. Isto implica em instabilidades para flutuações na densidade com cornprimentos de onda maiores que $\left|m_{J}\right|^{-1}$. Vale ressaltar que a inclusão de outras partículas internas como grávitons, bósons vetoriais ou férmions mudará este resultado apenas por uma constante ruitiplicativa $g$ em $\rho$ que conta os graus de liberdade destas particnlas. 


\section{Apêndice A}

Neste apêndice vamos obter as propriedades da furcção zeta de Riemann generalizada e calcular as integrais do tipo:

$$
J_{n}(t)=\left(\frac{i \pi T}{|\vec{k}|}\right)^{n-1} \int^{t} d y y^{n}\left[\ln (y)-\frac{1}{2 y}-\psi(y)\right]
$$

em termos da funçâo $\zeta$ definida como:

$$
\zeta(z, y)=\sum_{l=0}^{\infty} \frac{1}{(l+y)^{z}}
$$

Para isto, expressamas a funçāo $\psi$ coma:

$$
\xi(y)=\lim _{\varepsilon \rightarrow 0}\left[\frac{1}{\varepsilon}-\zeta(1+\varepsilon, y)\right]
$$

e usarnos a fórmula:

$$
\int^{t} d y(z, y)=\frac{1}{1-z} \zeta(1-z, t)
$$

que pode ser verificada facilmente pela equaça (A.2) e, integrando por partes, podz ser generalizada para:

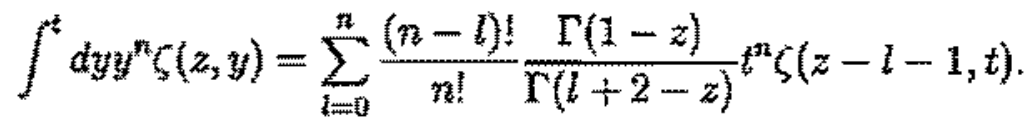

Substituindo a equação (A.3) na equação (A.1) e usando a equação (A.5) pode-se verificar que o terno divergente quando $\epsilon \rightarrow 0$ se cancela devido as 
propriedades da função $\zeta$, como era esperado. Para $n \neq 0$ obternos:

$$
\begin{aligned}
J_{n}(t)= & \left(\frac{i \pi T}{|\vec{k}|}\right)^{n-1}\left\{\frac{t^{n+1}}{n+1} \ln t-\frac{t^{n+1}}{(n+1)^{2}}-\frac{t^{n}}{2 n}-\right. \\
& -\sum_{j=0}^{n}(-1)^{j} t^{n-j}\left(\begin{array}{l}
n \\
j
\end{array}\right) \zeta^{t}(-j, t)+ \\
& \left.+\sum_{j=1}^{n} \frac{(-1)^{j}}{j+1}\left(\begin{array}{l}
n \\
j
\end{array}\right)\left(\sum_{k=1}^{j} \frac{1}{k}\right) t^{n-3} \mathrm{~B}_{j+1}(t)\right\}
\end{aligned}
$$

onde $\mathrm{B}_{n}$ são os polinônaios de Bernouli[11]. Para $J_{0}(t)$ obtemos:

$$
J_{0}(t)=\frac{|\vec{k}|}{\frac{k}{k} T} \int^{t}\left[\ln (y)-\frac{1}{2 y}-\psi(y)\right] d y=\frac{|\vec{k}|}{i \pi T}\left[t \ln t-\zeta^{\prime}(0, t)-t-\frac{1}{2} \ln t\right] .
$$

Vamos agora discutir o comportamento da funçäo zeta generalizada para pequenos valore do parâmetro $t\left(k_{0}\right)=i \frac{h_{0}+f(\hat{k})}{j \pi T}$, que corresponde ao limite de altas temperturas. Para isto, partimos da representaça 0 [11]:

$$
\zeta(z, t)=\frac{1}{z^{z}}+\frac{1}{\Gamma(z)} \int_{0}^{\infty} \frac{x^{z-1} e^{-k z}}{x^{x}-1} d x
$$

Expandindo (A.8) em série de potências de t e usando a representação integral da funçä́o gama de Euler, obtemos:

$$
\zeta(z, t)=\frac{1}{t^{z}}+\sum_{i=0}^{\infty} \frac{\Gamma(z+l)}{\Gamma(z)} \frac{(-t)^{l}}{l !} \zeta(z+l)
$$

Derivando (A.9) com relaçäo a $z_{1}$ obtemos, em termos da fançäo $\psi(z)$ que:

$$
\left.\zeta^{\prime}(z, t)=\sum_{l=0}^{\infty} \frac{(-t)^{l}}{l} \frac{\Gamma(z+l)}{\Gamma(z)}[\omega(z+l)-\psi(z)] \zeta(z+l)+\zeta^{\prime}(z+l)\right\}-t^{2} \ln (z)
$$

Estamos interessados realmente em valores de $\zeta^{\prime}(z, t)$ com $z \rightarrow-7$ onde $n$ è um damero inteiro positivo. Após um callculo sistematico, obternos: 


$$
\begin{aligned}
\zeta^{\prime}(-n, t)= & \sum_{i=0}^{n}\left(\begin{array}{c}
n \\
l
\end{array}\right)\left[\zeta(l-n)-\zeta(l-n) \sum_{k=n+1}^{n} \frac{1}{k}\right] t^{*}-t^{n} n(z) \\
& -\frac{t^{n+1}}{n+1}\left(\gamma-\sum_{k=1}^{n} \frac{1}{k}\right)+\sum_{i=n+2}^{\infty}(-1)^{n+1} \frac{n !(l-n-1) !}{l !} \zeta(l-n) .
\end{aligned}
$$

Desta forma, podemos expandir as funçöes de dois pontos calculadas neste trabalho en série decrescente de potências de $T$.

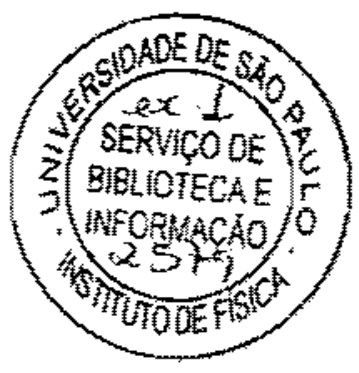




\section{Apêndice B}

Neste apêndice apresento as regras de Feynman para uma teoria de YangVills $S U(N)$ sern férmions no gauge de Feynman. Estas regras podem ser deduzidas pelo método usual de teoria quântica de campos a partir da densidade lagrangeana fetiva:

$$
\mathcal{L}_{\mathrm{pq}}=\mathcal{L}_{0}+\mathcal{L}_{\mathrm{FC}}+\mathcal{L}_{\mathrm{FP}}
$$

onde $\mathcal{L}_{0}$ ế a lagrangeana clássica dada por:

$$
\mathcal{L}_{\mathbb{0}}=\frac{1}{4} F^{a}{ }_{p D} F^{\mathrm{apur}}
$$

com $F$ dado por:

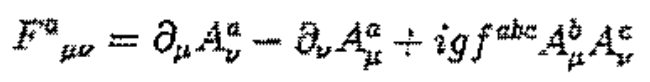

e $f$ é a constante de estrutura do grupo de gauge; 0 termo $\mathcal{L}_{\mathrm{FG}}$ em (B.I) corresponde ao termo de fixaçäo de gauge dado por:

$$
C_{\mathrm{FG}}=\frac{1}{2} \partial^{a} A_{j}^{a} \partial^{\mu} A_{\mu}^{a}
$$

e finalmente o termo $\mathcal{L}_{\text {Fp }}$ corresponde aos fantasmas de Fadeev-Popov:

$$
\mathcal{L}_{\mathrm{FP}}=\partial^{\mu} \bar{C}^{a}\left(\delta^{\mathrm{ab}} \partial_{\mu}+i g f^{a b c} A_{\mu}^{c}\right) \partial_{\mu} C
$$

Assira as regras de Feynman serẫo, para o propagador de um glion:

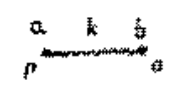

$$
\frac{\delta^{a b j} g_{\mu \omega}}{k^{2}}
$$


para o propagador de urn fantasma:

$$
\text { . k.... }
$$

para o vérice de três glưons:

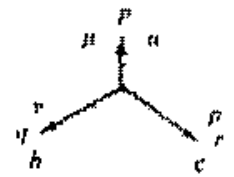

$$
\begin{aligned}
& g(2 \pi)^{4} \delta^{4}(p+q+r) f^{0.0}\left(g_{j w}(p-q)_{p}+\right. \\
& \left.+g_{\alpha p}(q-r)_{\alpha}+g_{p \alpha}(r-p)_{\nu}\right]
\end{aligned}
$$

para o vértice de quatro glúons:

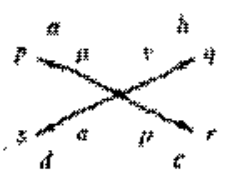

$$
\begin{aligned}
& g^{2}(2 \pi)^{4} b^{4}(p+q+r+s) \times
\end{aligned}
$$

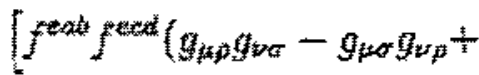

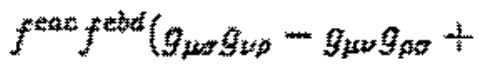

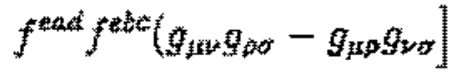

e para o vértice entre fantasmas e glúon:

$$
-g(2 \pi)^{d} \delta^{4}(p-q-k) f^{a b c} p_{\mu}
$$

lembrando de computar um sinal menos para cada loop de fantasma.

Para concluir este apêndice queria lembrat algumas propriedades da conș tante de estrutura do grupo de gauge SU(N):

$$
f^{a c d} f^{b a t}=N \delta^{a b}
$$

e

$$
f^{u d e} f^{b e f} f^{c f d}=N f^{a b c} .
$$

Desta forma podemos calcular os graficos de Feymman desta teoria 


\section{Bibliografia}

[1] E. Braten e R. D. Pisarski, Nucl. Phys. B337, 569 (1890); abid B339, 310 (1990); Phys. Rev. D_45, 1827 (1992).

[2] K. Kajantie e J. Kapusta, Ann. Phys. (NY) 160, 477 (1985)

[3] S. Mandelstam, Phys. Rev. D20, 3233 (1990)

[4] R. Jackiw e S. Ternpleton, Phys. Rev. D82, 615 (1981)

[5] A. P. de Alneida e J. Frenkel, Phys. Rev. D47, 640 (1993).

[6] A. P. de Almeida, F. T. C. Brandt e J. Frenkel, Phys. Rev. D49, 4196 (1994).

[7] J. I. Kapusta, Finite Temperature Field Theory (Cambridge University Press, Cambridge, 1989).

[8] N. D. Birrell e R. C. W. Davies, Quantum Field hn Curved Space (Cambridge University Press, 1982).

[9] S. Weinberg, Grazitation and Cosmology: Principles and Applications of the General Theory of Relativity (John Wiley, 1972)

[10] J. Frenkel e J. C. Taylor, Nucl. Phys. B334, 199 (1990); ibid B374, 156 (1992).

[11] I. S. Gradshteyn e I. M. Ryzhil, Tables of Integrals Series and Prodtcts (Academic, New York, 1980).

[12] A. P. Almeida, J. Frenkel e J. C. Taylor, Phys. Rev D 45, 2081 (1992) 
[13] A. D. Linde, Phys. Lett. B96, 289 (1980)

[14] O. K. Kalashwikov e V. V. Klimov, Fort. der Physik 31, 615 (1981)

[15] O. K. Kalashnikov, Fort. der Physik 32, 525 (1984)

[16] N. P. Landsman and Ch. G. van Weert, Phys. Rep. 145, 141 (1987). 